Pacific

Journal of

Mathematics

\title{
MULTIPLICATIVE DIRAC STRUCTURES
}

\section{CRISTIÁN ORTIZ}




\title{
MULTIPLICATIVE DIRAC STRUCTURES
}

\author{
CRISTIÁN ORTIZ
}

We introduce multiplicative Dirac structures on Lie groupoids, providing a unified framework to study both multiplicative Poisson bivectors (Poisson groupoids) and multiplicative closed 2-forms such as symplectic groupoids. We prove that for every source simply connected Lie groupoid $G$ with Lie algebroid $A G$, there exists a one-to-one correspondence between multiplicative Dirac structures on $G$ and Dirac structures on $A G$ that are compatible with both the linear and algebroid structures of $A G$. We explain in what sense this extends the integration of Lie bialgebroids to Poisson groupoids and the integration of Dirac manifolds. We explain the connection between multiplicative Dirac structures and higher geometric structures such as $\mathscr{L} A$ groupoids and $\mathscr{C} A$-groupoids.

1. Introduction 329

2. Tangent and cotangent structures 333

3. Multiplicative Dirac structures 339

4. Dirac algebroids 342

5. Infinitesimal description of multiplicative Dirac structures $\quad 345$

6. Conclusions and final remarks 362

Acknowledgements 362

References $\quad 362$

\section{Introduction}

Dirac structures were introduced by Courant and Weinstein [1988] as a common generalization of Poisson bivectors, closed 2-forms and regular foliations. A Dirac structure on a smooth manifold $M$ consists of a vector subbundle $L \subseteq \mathbb{T} M:=$ $T M \oplus T^{*} M$ that is maximal isotropic with respect to the nondegenerate symmetric pairing on $\mathbb{T} M$,

$$
\langle(X, \alpha),(Y, \beta)\rangle=\alpha(Y)+\beta(X),
$$

and that satisfies the integrability condition

$$
\llbracket \Gamma(L), \Gamma(L) \rrbracket \subseteq \Gamma(L),
$$

MSC2010: 53D17, 53D18.

Keywords: Lie groupoids, Lie algebroids, multiplicative Dirac structures. 
with respect to the Courant bracket $\llbracket \cdot, \cdot \mathbb{\|}: \Gamma(\mathbb{\square} M) \times \Gamma(\mathbb{}) \rightarrow \Gamma(\mathbb{}) M)$,

$$
\llbracket(X, \alpha),(Y, \beta) \rrbracket=\left([X, Y], \mathscr{L}_{X} \beta-i_{Y} d \alpha\right) .
$$

The integrability in the sense of Courant unifies different integrability conditions, including closed 2-forms, Poisson bivectors and regular foliations (see [Courant 1990b; Courant and Weinstein 1988]). More precisely, a 2-form $\omega$ on a smooth manifold $M$ induces a bundle map $\omega^{\sharp}: T M \rightarrow T^{*} M, X \mapsto \omega(X, \cdot)$ whose graph $L_{\omega}=\left\{\left(X, \omega^{\sharp}(X) \mid X \in T M\right)\right\}$ is a Lagrangian subbundle of $\mathbb{} \mathbb{}$. In this case, the Courant integrability of $L_{\omega}$ is equivalent to $\omega$ being a closed 2-form. Similarly, any bivector $\pi$ on $M$ defines a bundle map $\pi^{\sharp}: T^{*} M \rightarrow T M, \alpha \mapsto \pi(\alpha, \cdot)$ whose graph $L_{\pi}=\left\{\left(\pi^{\sharp}(\alpha), \alpha\right)\right\}$ is a Lagrangian subbundle of $\mathbb{} \pi$. One checks that $L_{\pi}$ satisfies the Courant integrability condition if and only if $\pi$ is a Poisson bivector. Also, if $F \subseteq T M$ is a regular subbundle we denote by $F^{\circ} \subseteq T^{*} M$ its annihilator. Then the Lagrangian subbundle $F \oplus F^{\circ} \subseteq \mathbb{}$ M is integrable in the sense of Courant if and only if $F \subseteq T M$ is involutive with respect to the Lie bracket of vector fields.

The main objective of this paper is to study Dirac structures defined on Lie groupoids, satisfying a suitable compatibility condition with the groupoid multiplication. Our study is motivated by a variety of geometrical structures compatible with group or groupoid structures, including:

(i) Poisson-Lie groups: A Poisson-Lie group is a Lie group $G$ with a Poisson structure $\pi$ that is compatible in the sense that the multiplication map $m: G \times G \rightarrow G$ is a Poisson map. Equivalently, the Poisson bivector $\pi$ is multiplicative, that is,

$$
\pi_{g h}=\left(l_{g}\right)_{*} \pi_{h}+\left(r_{h}\right)_{*} \pi_{g},
$$

for every $g, h \in G$. Here $l_{g}$ and $r_{h}$ denote the left and right multiplication by $g$ and $h$, respectively. Poisson-Lie groups arise as semiclassical limit of quantum groups, and they are infinitesimally described by Lie bialgebras. See for example, [Drinfel'd 1983].

(ii) Symplectic groupoids: A symplectic groupoid is a Lie groupoid $G$ with a symplectic structure $\omega$ that is compatible with the groupoid multiplication in the sense that the graph

$$
\operatorname{Graph}(m) \subseteq G \times G \times \bar{G}
$$

is a Lagrangian submanifold with respect to the symplectic structure $\omega \oplus \omega \ominus \omega$. This compatibility condition is equivalent to saying that $\omega$ is multiplicative, that is,

$$
m^{*} \omega=p r_{1}^{*} \omega+p r_{2}^{*} \omega,
$$

where $p r_{1}, p r_{2}: G_{(2)} \rightarrow G$ are the canonical projections and $G_{(2)} \subseteq G \times G$ is the set of composable groupoid pairs. Symplectic groupoids arise in the context of quantization of Poisson manifolds [Weinstein 1987; Weinstein and Xu 1991], 
connecting Poisson geometry to noncommutative geometry. In [Cattaneo and Felder 2001], symplectic groupoids appeared as phase spaces of certain sigma models. The infinitesimal description of symplectic groupoids is given by Poisson structures, see for example, [Weinstein 1987; Coste et al. 1987].

(iii) Poisson groupoids: These objects were introduced by A. Weinstein [1988] unifying Poisson-Lie groups and symplectic groupoids. A Poisson groupoid is a Lie groupoid $G$ equipped with a Poisson structure $\pi$ that is compatible with the groupoid multiplication in the sense that

$$
\operatorname{Graph}(m) \subseteq G \times G \times \bar{G}
$$

is a coisotropic submanifold. These structures are related to the geometry of the classical dynamical Yang-Baxter equation, see for instance [Etingof and Varchenko 1998]. At the infinitesimal level, Poisson groupoids are described by Lie bialgebroids [Mackenzie and Xu 1994].

(iv) Presymplectic groupoids: Lie groupoids equipped with a multiplicative closed 2 -form were studied in [Bursztyn et al. 2004]. A presymplectic groupoid is a Lie groupoid $G$ with a multiplicative closed 2-form $\omega$ satisfying suitable nondegeneracy conditions. These objects arise in connection with equivariant cohomology and generalized moment maps [Bursztyn and Crainic 2009]. The infinitesimal description of presymplectic groupoids is given by Dirac structures, extending the infinitesimal description of symplectic groupoids. More generally, Lie groupoids endowed with arbitrary multiplicative closed 2-forms are infinitesimally described by bundle maps $\sigma: A G \rightarrow T^{*} M$ called $I M$-2-forms. Here $A G$ denotes the Lie algebroid of $G$, and $T^{*} M$ is the cotangent bundle of the base of $G$.

The first goal of this work is to find a suitable definition of multiplicative Dirac structure that includes both multiplicative Poisson bivectors and multiplicative closed 2-forms, and hence encompasses all examples above. This is obtained by observing that given a Lie groupoid $G$ over $M$ with Lie algebroid $A G$, the tangent bundle $T G$ and the cotangent bundle $T^{*} G$ inherit natural Lie groupoid structures over $T M$ and $A^{*} G$, respectively. One observes that a bivector $\pi_{G}$ is multiplicative if and only if the bundle map $\pi_{G}^{\sharp}: T^{*} G \rightarrow T G$ is a groupoid morphism [Mackenzie and $\mathrm{Xu}$ 1994]. Similarly, a 2-form $\omega_{G}$ is multiplicative if and only if the bundle map $\omega_{G}^{\sharp}: T G \rightarrow T^{*} G$ is a morphism of Lie groupoids. It turns out that the direct sum vector bundle $T G \oplus T^{*} G$ is a Lie groupoid over $T M \oplus A^{*} G$, and graphs of both multiplicative Poisson bivectors and multiplicative closed 2-forms define Lie subgroupoids of $T G \oplus T^{*} G$. We say that a Dirac structure $L_{G}$ on a Lie groupoid $G$ is multiplicative if $L_{G} \subseteq T G \oplus T^{*} G$ is a Lie subgroupoid. A Lie groupoid $G$ equipped with a multiplicative Dirac structure is referred to as a Dirac groupoid. 
Our main purpose is to describe multiplicative Dirac structures infinitesimally, that is, in terms of Lie algebroid data. This work can be considered as a first step toward such a description. The main result of the present work says that for every source simply connected Lie groupoid $G$ with Lie algebroid $A G$, multiplicative Dirac structures on $G$ correspond to Dirac structures on $A G$ suitably compatible with both the linear and Lie algebroid structures on $A G$. In the particular case of multiplicative Poisson bivectors and multiplicative 2-forms, we explain how this is equivalent to the known infinitesimal descriptions carried out in [Mackenzie and $\mathrm{Xu}$ 2000] and [Bursztyn et al. 2004], respectively. Along the way, we develop techniques that can treat all multiplicative structures above in a unified manner, often simplifying existing results and proofs.

The present paper is organized as follows. In Section 2 we recall the definition of tangent and cotangent structures including tangent and cotangent groupoids and their algebroids, that is, tangent and cotangent algebroids. We also give an intrinsic construction of the tangent lift of a Dirac structure, providing an alternative proof of the results shown in [Courant 1990a]. In Section 3 we define the main objects of our study, multiplicative Dirac structures. We discuss a variety of examples arising in nature, including foliated groupoids, Dirac Lie groups, tangent lifts of multiplicative Dirac structures, symmetries of multiplicative Dirac structures (for example, reduction of Poisson groupoids), $B$-field transformations of multiplicative Dirac structures and generalized complex groupoids. In Section 4 we introduce the notion of Dirac algebroid and also several examples are discussed, including foliated algebroids, Dirac Lie algebras, tangent lifts of Dirac algebroids, symmetries of Dirac algebroids (for example, reduction of Lie bialgebroids), $B$-field transformations of Dirac algebroids and generalized complex algebroids. In Section 5 we explain how the multiplicativity of a Dirac structure is reflected at the Lie algebroid level, proving the main result of this work, which says that if $G$ is a source simply connected Lie groupoid with Lie algebroid $A G$, then there is a one-to-one correspondence between Dirac groupoid structures on $G$ and Dirac algebroid structures on $A G$. Along the way, we explain the relation between multiplicative Dirac structures and higher structures such as $\mathscr{C} \mathscr{A}$-groupoids and $\mathscr{L} \mathscr{A}$-groupoids. We also relate the examples of Section 3 with the examples of Section 4, in the spirit of the correspondence established by the main result of the paper. In Section 6, we discuss conclusions and work in progress.

1A. Notation and conventions. For a Lie groupoid $G$ over $M$ we denote by $s, t$ : $G \rightarrow M$ the source and target maps, respectively. The multiplication map is denoted by $m: G_{(2)} \rightarrow G$, where $G_{(2)}=\{(g, h) \in G \times G \mid s(g)=t(h)\}$ is the set of composable pairs. The Lie algebroid of $G$ is defined by $A G:=\left.\operatorname{Ker}(T s)\right|_{M}$, with Lie bracket given by identifying sections of $A G$ with right-invariant vector 
fields on $G$ and anchor map $\rho_{A G}:=\left.T t\right|_{A G}: A G \rightarrow T M$. Given a Lie groupoid morphism $\Psi: G_{1} \rightarrow G_{2}$, the corresponding Lie algebroid morphism is denoted by $A(\Psi): A G_{1} \rightarrow A G_{2}$. Arbitrary Lie algebroids are denoted by $A \rightarrow M$ with Lie bracket $[\cdot, \cdot]_{A}$ and anchor map $\rho_{A}$. Also, given a smooth manifold $M$, the tangent bundle is denoted by $p_{M}: T M \rightarrow M$ and the cotangent bundle is denoted by $c_{M}: T^{*} M \rightarrow M$.

\section{Tangent and cotangent structures}

2A. Tangent and cotangent groupoids. Let $G$ be a Lie groupoid over $M$ with Lie algebroid $A G$. The tangent bundle $T G$ has a natural Lie groupoid structure over $T M$. This structure is obtained by applying the tangent functor to each of the structure maps defining $G$ (source, target, multiplication, inversion and identity section). We refer to $T G$ with this groupoid structure over $T M$ as the tangent groupoid of $G$. The set of composable pairs of $T G$ is $(T G)_{(2)}=T\left(G_{(2)}\right)$, and for $(g, h) \in G_{(2)}$ and a tangent groupoid pair $\left(X_{g}, Y_{h}\right) \in(T G)_{(2)}$ the multiplication map on $T G$ is

$$
X_{g} \bullet Y_{h}:=\operatorname{Tm}\left(X_{g}, Y_{h}\right) .
$$

Now consider the cotangent bundle $T^{*} G$. It was shown in [Coste et al. 1987] that $T^{*} G$ is a Lie groupoid over $A^{*} G$. The source and target maps are defined by

$$
\tilde{s}\left(\alpha_{g}\right) u=\alpha_{g}\left(T l_{g}(u-T t(u))\right) \quad \text { and } \quad \tilde{t}\left(\beta_{g}\right) v=\beta_{g}\left(\operatorname{Tr}_{g}(v)\right) \text {, }
$$

where $\alpha_{g} \in T_{g}^{*} G, u \in A_{s(g)} G$ and $\beta_{g} \in T_{g}^{*} G, v \in A_{t(g)} G$. The multiplication on $T^{*} G$ is defined by

$$
\left(\alpha_{g} \circ \beta_{h}\right)\left(X_{g} \bullet Y_{h}\right)=\alpha_{g}\left(X_{g}\right)+\beta_{h}\left(Y_{h}\right)
$$

for $\left(X_{g}, Y_{h}\right) \in T_{(g, h)} G_{(2)}$.

We refer to $T^{*} G$ with the groupoid structure over $A^{*} G$ as the cotangent groupoid of $G$.

2B. Tangent and cotangent algebroids. Let $q_{A}: A \rightarrow M$ be a vector bundle over $M$. The tangent bundle $T A$ has a natural structure of a double vector bundle [Pradines 1974], given by the diagram below.

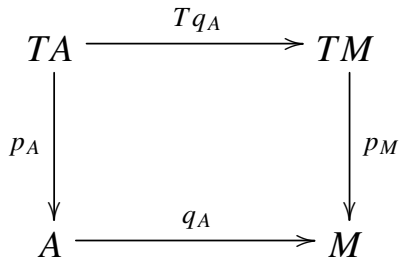


Assume now that $q_{A}: A \rightarrow M$ has a Lie algebroid structure with anchor map $\rho_{A}: A \rightarrow T M$ and Lie bracket $[\cdot, \cdot]$ on $\Gamma_{M}(A)$.

As explained in [Mackenzie 2005], there is a canonical Lie algebroid structure on the vector bundle $T q_{A}: T A \rightarrow T M$. Recall that there exists a canonical involution $J_{M}: T T M \rightarrow T T M$, which is a morphism of double vector bundles. In a local coordinates system $\left(x^{i}, \dot{x}^{i}, \delta x^{i}, \delta \dot{x}^{i}\right)$ on $T T M$ this map is given by

$$
J_{M}\left(\left(x^{i}, \dot{x}^{i}, \delta x^{i}, \delta \dot{x}^{i}\right)\right)=\left(x^{i}, \delta x^{i}, \dot{x}^{i}, \delta \dot{x}^{i}\right) .
$$

Notice that the map $J_{M}$ yields a vector bundle isomorphism as below.

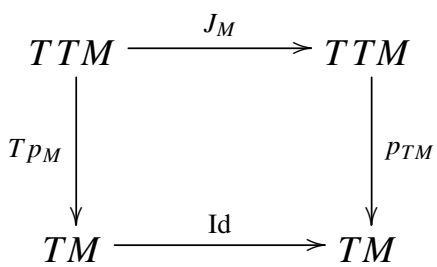

Now we can apply the tangent functor to the anchor map $\rho_{A}: A \rightarrow T M$, yielding a bundle map $T \rho_{A}: T A \rightarrow T T M$, where $T T M$ is equipped with bundle projection $T p_{M}: T T M \rightarrow T M$. Therefore, composing $T \rho_{A}$ with the canonical involution $J_{M}$ we obtain the bundle map $\rho_{T A}: T A \rightarrow T T M$, defined by

$$
\rho_{T A}:=J_{M} \circ T \rho_{A},
$$

which is a vector bundle morphism from $T A \rightarrow T T M$, where the target bundle is the one corresponding to the usual bundle projection $p_{T M}: T T M \rightarrow T M$. The map $\rho_{T A}: T A \rightarrow T T M$, as above, defines the tangent anchor map. In order to define the tangent Lie bracket, we observe that every section $u \in \Gamma_{M}(A)$ induces two types of sections of $T q_{A}: T A \rightarrow T M$. The first type corresponds to the linear section $T u: T M \rightarrow T A$, which is given by applying the tangent functor to the section $u: M \rightarrow A$. The second type of section is the core section $\hat{u}: T M \rightarrow T A$, which is defined by

$$
\hat{u}(X)=T\left(0^{A}\right)(X)+{ }_{A} \overline{u\left(p_{M}(X)\right)},
$$

where $0^{A}: M \rightarrow A$ denotes the zero section, and $\overline{u\left(p_{M}(X)\right)}=d /\left.d t\left(t u\left(p_{M}(X)\right)\right)\right|_{t=0}$. As observed in [Mackenzie and Xu 1994], sections of the form $T u$ and $\hat{u}$ generate the module of sections $\Gamma_{T M}(T A)$. Therefore, the tangent Lie bracket is determined by

$$
[T u, T v]=T[u, v], \quad[T u, \hat{v}]=\widehat{[u, v]}, \quad[\hat{u}, \hat{v}]=0,
$$

and we extend to other sections by requiring the Leibniz rule with respect to the tangent anchor $\rho_{T A}$. This defines the natural Lie algebroid structure on $T q_{A}: T A \rightarrow$ $T M$. 
Example 2.1. Assume that $A=\mathfrak{g}$ is a Lie algebra, that is, a Lie algebroid over a point. In this case, the tangent Lie algebra structure on $T \mathfrak{g}=\mathfrak{g} \times \mathfrak{g}$ is given by the semidirect product Lie algebra determined by the adjoint representation of $\mathfrak{g}$ on itself.

Following [Mackenzie 2005], the cotangent bundle of a Lie algebroid inherits a Lie algebroid structure. For that, let us explain the vector bundle structure $T^{*} A \rightarrow A^{*}$. If $\left(x^{i}, u^{a}\right)$ are local coordinates on $A$, we induce a local coordinates system $\left(x^{i}, u^{a}, p_{i}, \lambda_{a}\right)$ on $T^{*} A$, where $\left(p_{i}\right)$ determines a cotangent element in $T_{x}^{*} M$ and $\left(\lambda_{a}\right) \in A_{x}^{*}$ is a cotangent element with respect to the tangent direction to the fibers of $A$. Now the bundle projection $r: T^{*} A \rightarrow A^{*}$ is described locally by $r\left(x^{i}, u^{a}, p_{i}, \lambda_{a}\right)=\left(x^{i}, \lambda_{a}\right)$. These vector bundle structures define a commutative diagram

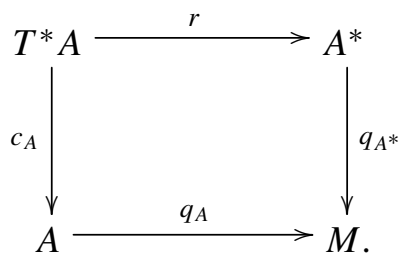

This endows $T^{*} A$ with a double vector bundle structure. Suppose that $q_{A}: A \rightarrow M$ carries a Lie algebroid structure. Then we can consider the dual bundle $A^{*}$ endowed with the linear Poisson structure induced by $A$. The cotangent bundle $T^{*} A^{*} \rightarrow A^{*}$ has the Lie algebroid structure determined by the linear Poisson bivector on $A^{*}$. There exists a Legendre type map $R: T^{*} A^{*} \rightarrow T^{*} A$ that is an antisymplectomorphism with respect to the canonical symplectic structures, and it is locally defined by $R\left(x^{i}, \xi_{a}, p_{i}, u^{a}\right)=\left(x^{i}, u^{a},-p_{i}, \xi_{a}\right)$. For an intrinsic definition see [Mackenzie and Xu 1994; Tulczyjew 1977].

Definition 2.2. The cotangent algebroid of $A$ is the vector bundle $T^{*} A \rightarrow A^{*}$ equipped with the unique Lie algebroid structure that makes the Legendre type transform $R: T^{*}\left(A^{*}\right) \rightarrow T^{*} A$ into an isomorphism of Lie algebroids.

Example 2.3. Suppose that $A=\mathfrak{g}$ is a Lie algebra, that is, a Lie algebroid over a point. Then, the cotangent algebroid $T^{*} \mathfrak{g}=\mathfrak{g} \times \mathfrak{g}^{*} \rightarrow \mathfrak{g}^{*}$ is given by the transformation Lie algebroid with respect to the coadjoint representation of $\mathfrak{g}$ on its dual vector space $\mathfrak{g}^{*}$.

Finally, recall also that the Tulczyjew map $\Theta_{M}: T T^{*} M \rightarrow T^{*} T M$ is the isomorphism which, in a local coordinates system $\left(x^{i}, p_{i}, \dot{x}^{i}, \dot{p}_{i}\right)$, is given by

$$
\Theta_{M}\left(x^{i}, p_{i}, \dot{x}^{i}, \dot{p}_{i}\right)=\left(x^{i}, \dot{x}^{i}, \dot{p}_{i}, p_{i}\right) .
$$

See [Mackenzie and Xu 1994; Tulczyjew 1977] for an intrinsic definition. Consider now a Lie groupoid $G$ over $M$ with Lie algebroid $A G=\left.\operatorname{ker} T s\right|_{M}$. There 
exists a natural injective bundle map

$$
i_{A G}: A G \rightarrow T G .
$$

The canonical involution $J_{G}: T T G \rightarrow T T G$ restricts to an isomorphism of Lie algebroids $j_{G}: T(A G) \rightarrow A(T G)$. More precisely, there exists a commutative diagram

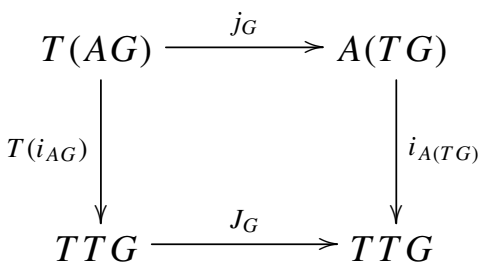

In particular, the Lie algebroid $A(T G)$ of the tangent groupoid is canonically isomorphic to the tangent Lie algebroid $T(A G)$ of $A G$. Similarly, the Lie algebroid of the cotangent groupoid $T^{*} G$ is isomorphic to the cotangent Lie algebroid $T^{*}(A G)$. For that, notice that the natural pairing $T^{*} G \oplus T G \rightarrow \mathbb{R}$ defines a groupoid morphism, and the application of the Lie functor yields a symmetric pairing $\left\langle\langle\cdot, \cdot\rangle: A\left(T^{*} G\right) \oplus\right.$ $A(T G) \rightarrow \mathbb{R}$, which is nondegenerate. See for example, [Mackenzie and Xu 1994; 2000]. In particular, we obtain an isomorphism $K_{G}: A\left(T^{*} G\right) \rightarrow A(T G)^{*}$, where the target dual is with respect to the fibration $A(T G) \stackrel{A\left(p_{G}\right)}{\longrightarrow} A G$. Now we define a Lie algebroid isomorphism

$$
j_{G}^{\prime}: A\left(T^{*} G\right) \rightarrow T^{*}(A G),
$$

determined by the composition $j_{G}^{\prime}=j_{G}^{*} \circ K_{G}$, where $j_{G}^{*}: A(T G)^{*} \rightarrow T^{*}(A G)$ is the bundle map dual to the isomorphism $j_{G}: T(A G) \rightarrow A(T G)$. As $j_{G}: T(A G) \rightarrow$ $A(T G)$ is a suitable restriction of the canonical involution $J_{G}: T T G \rightarrow T T G$, the isomorphism $j_{G}^{\prime}$ is related to the Tulczyjew map $\Theta_{G}: T T^{*} G \rightarrow T^{*} T G$, via

$$
j_{G}^{\prime}=\left(T i_{A G}\right)^{*} \circ \Theta_{G} \circ i_{A\left(T^{*} G\right)} .
$$

2C. Tangent lift of a Dirac structure. The tangent lift of Dirac structures was originally studied by T. Courant [1990a], who described tangent Dirac structures locally. I. Vaisman [2005] gives an intrinsic construction of tangent Dirac structures, where the tangent lift of a Dirac structure is described via the sheaf of local sections defining a Dirac subbundle of $T T M \oplus T^{*} T M$. Here, we provide an alternative description of the tangent lift of a Dirac structure relied on the tangent lift of Lie algebroid structures described in the previous section.

In order to fix our notation, we begin by summarizing some of the main properties of tangent lifts of vector fields and differential forms, see [Grabowski and Urbański 1997; Yano and Ishihara 1973]. Let $f \in C^{\infty}(M)$ be a smooth function. Then we 
have a pair of smooth functions on $T M$ defined by

$$
f^{v}=f \circ p_{M}, \quad f^{T}=d f .
$$

We refer to $f^{v}$ and $f^{T}$ as the vertical and tangent lifts of $f$. One can see easily that the algebra of functions $C^{\infty}(T M)$ is generated by functions of the form $f^{v}$ and $f^{T}$. Now, given a vector field $X$ on $M$ we define the vertical lift of $X$ as the vector field $X^{v}$ on $T M$ that acts on vertical and tangent lifts of functions as

$$
X^{v}\left(f^{v}\right)=0, \quad X^{v}\left(f^{T}\right)=(X f)^{v} .
$$

The tangent lift of $X$ is the vector field $X^{T}$ on $T M$ that acts on vertical and tangent lifts of functions in the following manner:

$$
X^{T}\left(f^{v}\right)=(X f)^{v}, \quad X^{T}\left(f^{T}\right)=(X f)^{T} .
$$

It is easy to see that vertical and tangent lifts of vector fields generate the space of all vector fields on $T M$. Now let us consider a 1 -form $\alpha$ on a smooth manifold $M$. We define the vertical lift of $\alpha$ as the 1-form $\alpha^{v}$ on $T M$, which is determined by its value at vertical and tangent lifts of vector fields,

$$
\alpha^{v}\left(X^{v}\right)=0, \quad \alpha^{v}\left(X^{T}\right)=(\alpha(X))^{v} .
$$

The tangent lift of $\alpha$ is the 1 -form $\alpha^{T}$ on $T M$ defined by

$$
\alpha^{T}\left(X^{v}\right)=(\alpha(X))^{v}, \quad \alpha^{T}\left(X^{T}\right)=(\alpha(X))^{T} .
$$

It is important to emphasize that vertical and tangent lifts of vector fields (resp. of 1-forms) are sections of the usual vector bundle structure $T(T M) \stackrel{p_{T M}}{\longrightarrow} T M$ (resp. sections of $T^{*}(T M) \stackrel{c_{T M}}{\longrightarrow} T M$ ), and they do not define sections of the tangent prolongation vector bundle $T(T M) \stackrel{T p_{M}}{\longrightarrow} T M$ (resp. of the tangent prolongation $\left.T\left(T^{*} M\right) \stackrel{T c_{M}}{\longrightarrow} T M\right)$. However, there exists a canonical relation between vector fields (resp. 1-forms) on $T M$ and sections of the tangent prolongation vector bundle $T(T M) \rightarrow T M$ (resp. $T\left(T^{*} M\right) \rightarrow T M$ ). Given a vector field $X$ and a 1-form $\alpha$ on $M$, we consider the linear sections $T X, T \alpha$ and the core sections $\hat{X}, \hat{\alpha}$ of the corresponding tangent prolongation vector bundles. It follows from the definition that

$$
\begin{aligned}
J_{M}(T X)=X^{T}, & J_{M}(\hat{X})=X^{v} . \\
\Theta_{M}(T \alpha)=\alpha^{T}, & \Theta_{M}(\hat{\alpha})=\alpha^{v} .
\end{aligned}
$$

It turns out that many geometric properties of the direct sum vector bundle $T(T M) \oplus$ $T^{*}(T M)$ can be understood in terms of tangent geometric properties of $T(T M) \oplus$ $T\left(T^{*} M\right)$, using the canonical identification

$$
J_{M} \oplus \Theta_{M}: T(T M) \oplus T\left(T^{*} M\right) \rightarrow T(T M) \oplus T^{*}(T M) .
$$


Now consider a Dirac structure $L_{M}$ on $M$. Equivalently, we may think of $L_{M}$ as a Lie algebroid over $M$ with Lie bracket given by the Courant bracket on sections of $L_{M}$, and the anchor map $\rho_{M}$ is the natural projection from $L_{M} \subseteq T M \oplus T^{*} M$ onto TM. According to a construction of K. Mackenzie and P. Xu [1994], we can consider the tangent prolongation Lie algebroid $T L_{M} \rightarrow T M$, with anchor map

$$
\rho_{T M}=J_{M} \circ T \rho_{M},
$$

and Lie bracket defined by

$$
\left.\left[\hat{a}_{1}, \hat{a}_{2}\right]_{T L_{M}}=0, \quad\left[T a_{1}, \hat{a}_{2}\right]_{T L_{M}}=\widehat{\left[a_{1}, a_{2}\right.}\right], \quad\left[T a_{1}, T a_{2}\right]_{T L_{M}}=T\left[a_{1}, a_{2}\right],
$$

where $a_{1}, a_{2}$ are sections of $L_{M} \rightarrow M$. We denote by $L_{T M}$ the image of $T L_{M}$ under the natural bundle map $J_{M} \oplus \Theta_{M}: T T M \oplus T T^{*} M \rightarrow T T M \oplus T^{*} T M$.

Proposition 2.4. The subbundle $L_{T M} \subseteq T T M \oplus T^{*} T M$ is isotropic with respect to the nondegenerate symmetric pairing $\langle\cdot, \cdot\rangle_{T M}$ defined on TTM $\oplus T^{*} T M$.

Proof. Let $(X, \alpha),(Y, \beta)$ be sections of $L_{M}$. Then, the tangent lifts $\left(X^{T}, \alpha^{T}\right)$ and $\left(Y^{T}, \beta^{T}\right)$ define sections of $L_{T M}$. Notice that

$$
\left\langle\left(X^{T}, \alpha^{T}\right),\left(Y^{T}, \beta^{T}\right)\right\rangle=(\beta(X))^{T}+(\alpha(Y))^{T}=(\langle(X, \alpha),(Y, \beta)\rangle)^{T}=0 .
$$

This implies that $L_{T M}$ is isotropic.

The tangent Lie algebroid $T L_{M} \rightarrow T M$ induces a unique Lie algebroid structure on $L_{T M} \rightarrow T M$ characterized by the property that $J_{M} \oplus \Theta_{M}: T L_{M} \rightarrow L_{T M}$ is a Lie algebroid isomorphism. The space of sections $\Gamma\left(L_{T M}\right)$ is generated by sections of the form $a^{T}:=\left(J_{M} \oplus \Theta_{M}\right)(T a)$ and $a^{v}:=\left(J_{M} \oplus \Theta_{M}\right) \hat{a}$, where $a$ is a section of $L_{M} \rightarrow M$. In particular the induced Lie bracket on sections of $L_{T M}$ is completely determined by identities

$$
\left[a_{1}^{v}, a_{2}^{v}\right]=0, \quad\left[a_{1}^{T}, a_{2}^{v}\right]=\llbracket a_{1}, a_{2} \rrbracket^{v}, \quad\left[a_{1}^{T}, a_{2}^{T}\right]=\llbracket a_{1}, a_{2} \rrbracket^{T},
$$

and the Leibniz rule with respect to the induced anchor map $\operatorname{pr}_{T T M}: L_{T M} \rightarrow T T M$.

Proposition 2.5. The induced Lie bracket on sections $\Gamma\left(L_{T M}\right)$ is a restriction of the Courant bracket $\mathbb{[} \cdot, \cdot \mathbb{1}_{T M}$ on sections of TTM $\oplus T^{*} T M$.

Proof. Due to the identities (7) and (8), we only need to check that the Courant bracket on sections of $L_{T M}$, naturally induced by $J_{M} \oplus \Theta_{M}$, satisfies the bracket identities that determine the induced Lie bracket on $\Gamma\left(L_{T M}\right)$. One observes that vertical and tangent lifts are compatible with Lie derivatives in the sense that

(1) $\mathscr{L}_{X^{v}} \alpha^{v}=0$,

(2) $\mathscr{L}_{X^{T}} \alpha^{v}=\left(\mathscr{L}_{X} \alpha\right)^{v}$,

(3) $\mathscr{L}_{X^{T}} \alpha^{T}=\left(\mathscr{L}_{X} \alpha\right)^{T}$, 
and we conclude that

(1) $\llbracket X^{v} \oplus \alpha^{v}, Y^{v} \oplus \beta^{v} \rrbracket=0$,

(2) $\llbracket X^{T} \oplus \alpha^{T}, Y^{v} \oplus \beta^{v} \rrbracket=[X, Y]^{v} \oplus\left(\mathscr{L}_{X} \beta-i_{Y} d \alpha\right)^{v}$,

(3) $\llbracket X^{T} \oplus \alpha^{T}, Y^{T} \oplus \beta^{T} \rrbracket=[X, Y]^{T} \oplus\left(\mathscr{L}_{X} \beta-i_{Y} d \alpha\right)^{T}$.

Thus the Lie bracket on $\Gamma_{T M}\left(L_{T M}\right)$ induced by the tangent Lie bracket on $\Gamma_{T M}\left(T L_{M}\right)$ coincides with the Courant bracket.

We have shown the following:

Proposition 2.6. Let $M$ be a smooth manifold. There exists a natural map

$$
\begin{aligned}
\operatorname{Dir}(M) & \rightarrow \operatorname{Dir}(T M) \\
L_{M} & \mapsto L_{T M},
\end{aligned}
$$

where $L_{T M}:=\left(J_{M} \oplus \Theta_{M}\right)\left(T L_{M}\right)$.

The Dirac structure $L_{T M} \in \operatorname{Dir}(T M)$ given by the proposition above is referred to as the tangent Dirac structure induced by $L_{M} \in \operatorname{Dir}(M)$. It is straightforward to check that this construction unifies the tangent lift of both closed 2-forms and Poisson bivectors. Additionally, the presymplectic foliation of $L_{T M}$ corresponds to taking the tangent bundle of each leaf endowed with the tangent lift of the leafwise presymplectic forms defined by $L_{M}$. See also [Boumaiza and Zaalani 2009] for a general construction of tangent lifts of Dirac structures on arbitrary Courant algebroids.

\section{Multiplicative Dirac structures}

This section introduces the main objects of study of this work, that is, Lie groupoids equipped with Dirac structures compatible with the groupoid multiplication, including both multiplicative Poisson and closed 2-forms as particular cases.

3A. Definition and main examples. Let $G$ be a Lie groupoid over $M$, with Lie algebroid $A G$. Consider the direct sum Lie groupoid $\mathbb{T} G=T G \oplus T^{*} G$ with base manifold $T M \oplus A^{*} G$.

Definition 3.1. Let $G$ be a Lie groupoid over $M$. A Dirac structure $L_{G}$ on $G$ is said to be multiplicative if $L_{G} \subseteq T G \oplus T^{*} G$ is a subgroupoid over some subbundle $E \subseteq T M \oplus A^{*} G$.

We refer to a pair $\left(G, L_{G}\right)$, made up of a Lie groupoid $G$ and a multiplicative Dirac structure $L_{G}$ on $G$, as a Dirac groupoid. We use the notation $\operatorname{Dir}_{\text {mult }}(G)$ to indicate the set consisting of all multiplicative Dirac structures on $G$.

It follows from the multiplicativity of $L_{G}$ that $E \subseteq T M \oplus A^{*} G$ is a vector subbundle. In particular, a multiplicative Dirac structure $L_{G}$ on a Lie groupoid $G$ defines a $\mathscr{V} \mathscr{B}$-subgroupoid $L_{G} \subseteq \mathbb{T} G$. 
Example 3.2. Let $\omega_{G}$ be a closed multiplicative 2-form on a Lie groupoid $G$. The multiplicativity property of $\omega_{G}$ is equivalent to saying that the bundle map $\omega_{G}^{\sharp}: T G \rightarrow T^{*} G$ is a morphism of Lie groupoids. Hence, the corresponding Dirac structure $L_{\omega_{G}}=\operatorname{Graph}\left(\omega_{G}\right) \subseteq \mathbb{T} G$ is a multiplicative Dirac structure. In this case we have a groupoid $L_{\omega_{G}} \rightrightarrows E$, where $E \subseteq T M \oplus A^{*} G$ is the subbundle given by the graph of the bundle map $-\sigma^{t}$ determined by the IM-2-form (see [Bursztyn et al. 2004]) $\sigma$ associated to $\omega_{G}$.

Example 3.3. Let $\left(G, \pi_{G}\right)$ be a Poisson groupoid. The multiplicativity of $\pi_{G}$ is equivalent to saying that $\pi_{G}^{\sharp}: T^{*} G \rightarrow T G$ is a morphism of Lie groupoids. Therefore, the associated Dirac structure $L_{\pi_{G}}=\operatorname{Graph}\left(\pi_{G}\right) \subseteq \mathbb{T} G$ defines a multiplicative Dirac structure. In this case we have a groupoid $L_{\pi_{G}} \rightrightarrows E$, where $E \subseteq T M \oplus A^{*} G$ is the subbundle given by the graph of dual anchor map $\rho_{A^{*} G}: A^{*} G \rightarrow T M$.

The examples discussed previously show that Dirac groupoids lead to a natural generalization of Poisson groupoids and presymplectic groupoids. Our main aim is to describe Dirac groupoids infinitesimally, establishing in particular, a connection between such an infinitesimal description and Lie bialgebroids and IM-2-forms.

3B. More examples of multiplicative Dirac structures. In addition to multiplicative closed 2-forms and multiplicative Poisson bivectors, there are several interesting multiplicative Dirac structures, which will be discussed throughout this subsection.

3B1. Foliated groupoids. A regular distribution $F_{G} \subseteq T G$ is called multiplicative if it defines a Lie subgroupoid of the tangent groupoid $T G$. A foliated groupoid is a pair $\left(G, F_{G}\right)$, where $G$ is a Lie groupoid and $F_{G}$ is an involutive multiplicative regular distribution. In this case, the Dirac structure $F_{G} \oplus F_{G}^{\circ} \subseteq \mathbb{T} G$ is easily seen to be a multiplicative Dirac structure on $G$. The foliation tangent to an involutive multiplicative distribution is called a multiplicative foliation. Multiplicative foliations that are simultaneously transversal to the $s$-fibration and to the $t$-fibration were studied in [Tang 2006], providing interesting examples of noncommutative Poisson algebras. Also, multiplicative foliations arise in the context of geometric quantization of symplectic groupoids, namely, as polarizations compatible with a symplectic groupoid structure (see [Hawkins 2008]). In addition, the notion of multiplicative foliation has appeared in connection with exterior differential systems. For more details see [Crainic et al. 2012] and the references therein.

3B2. Dirac Lie groups. Dirac Lie groups, that is, Lie groups equipped with multiplicative Dirac structures, were first studied by the author in [Ortiz 2008], providing a generalization of Poisson Lie groups within the category of Lie groups. In that work, it is shown that, modulo regularity issues, Dirac Lie groups are given by the pull-back (in the sense of Dirac structures) of Poisson Lie groups via a surjective submersion which is also a Lie group morphism. Notice that whenever a Lie 
groupoid $G$ over $M$ is equipped with a multiplicative Dirac structure, then for every $x \in M$, the isotropy Lie group $G_{x}:=s^{-1}(x) \cap t^{-1}(x)$ inherits a Dirac structure $L_{G_{x}}$ making the pair $\left(G_{x}, L_{G_{x}}\right)$ into a Dirac Lie group.

We emphasize that different notions of Dirac Lie groups exist in the literature. For instance, Li-Bland and Meinrenken [2011] have proposed a notion of multiplicativity that includes interesting examples of twisted Dirac structures on Lie groups such as the Cartan-Dirac structure on a compact Lie group.

3B3. Tangent lift of a multiplicative Dirac structure. In [Grabowski and Urbański $1995]$ it was proved that whenever a Lie group $G$ carries a multiplicative Poisson bivector $\pi_{G}$, then the tangent Lie group $T G$ equipped with the tangent Poisson structure $\pi_{T G}$ becomes a Poisson Lie group. It is easy to extend the multiplicative Poisson case to abstract multiplicative Dirac structures on Lie groupoids. Assume that $G$ is a Lie groupoid over $M$ and consider the tangent groupoid $T G$ over $T M$ explained in Section 2A. Then, the tangent Dirac structure $L_{T G} \subseteq T T G \oplus T^{*} T G$ induced by a multiplicative Dirac structure $L_{G} \subseteq T G \oplus T^{*} G$ is also a multiplicative Dirac structure. Indeed, first observe that the bundle map $J_{G}: T T G \rightarrow T T G$ is a groupoid isomorphism over $J_{M}: T T M \rightarrow T T M$. Similarly, the bundle map $\Theta_{G}: T T^{*} G \rightarrow T^{*} T G$ is a groupoid isomorphism over the canonical identification $I: T\left(A^{*} G\right) \rightarrow(T(A G))^{*}$. Since $L_{G}$ is a Lie subgroupoid of $T G \oplus T^{*} G$, then the tangent functor yields a Lie subgroupoid $T L_{G}$ of $T T G \oplus T T^{*} G$. Due to the fact that $L_{T G}$ is the image of $T L_{G}$ via the groupoid isomorphism $J_{G} \oplus \Theta_{G}$, we see that $L_{T G}$ inherits a natural structure of Lie subgroupoid of $T T G \oplus T^{*} T G$. Hence we conclude that $L_{T G}$ defines a multiplicative Dirac structure on $T G$.

3B4. Symmetries of multiplicative Dirac structures. Let $L_{G}$ be a multiplicative Dirac structure on a Lie groupoid $G \rightrightarrows M$, and let $H$ be a Lie group acting on $G$ by groupoid automorphisms. Assume that the $H$-action is free and proper and that the $H$-orbits coincide with the characteristic leaves of $L_{G}$. In this case the quotient space $G / H$ inherits the structure of a Lie groupoid over $M / H$. Moreover, since $G / H$ is the space of characteristic leaves of $L_{G}$, we conclude that there exists a Poisson structure $\pi_{\text {red }}$ on $G / H$, making the quotient map $G \rightarrow G / H$ into both a backward and forward Dirac map. This fact together with the multiplicativity of $L_{G}$ imply that $\pi_{\text {red }}$ is a multiplicative Poisson bivector. In other words, the quotient space $G / H$ is a Poisson groupoid. In the case where $L_{G}$ is the graph of a multiplicative Poisson bivector and the action is Hamiltonian in the sense of Fernandes and Iglesias [ $\geq 2013]$, this recovers some of the results about reduction of Poisson groupoids carried out by those authors.

3B5. Multiplicative B-field transformations. Let $L \subseteq \mathbb{\mathbb { M }}$ be a Lagrangian subbundle. Given a 2-form $B \in \Omega^{2}(M)$ one can construct the Lagrangian subbundle 
$\tau_{B}(L) \subseteq \mathbb{\square} M$ defined by

$$
\tau_{B}(L)=\left\{X \oplus \alpha+i_{X} B \mid X \oplus \alpha \in L\right\} .
$$

A straightforward computation shows that $\tau_{B}(L)$ defines a Dirac structure on $M$ if and only if $B$ is a closed 2-form. See for instance [Bursztyn 2005; Gualtieri 2003]. In this case, we say that the Dirac structure $\tau_{B}(L)$ is obtained out of $L$ by a $B$-field transformation.

Assume now that $L_{G}$ is a multiplicative Dirac structure on a Lie groupoid $G$. Given a multiplicative closed 2-form $B_{G}$ on $G$, one can consider the bundle map $\tau_{B_{G}}: \mathbb{T} G \rightarrow \mathbb{T} G, X \oplus \alpha \mapsto X \oplus \alpha+i_{X}\left(B_{G}\right)$. It follows from the multiplicativity of $B_{G}$ that $\tau_{B_{G}}$ is a Lie groupoid isomorphism. As a result, the Dirac structure $\tau_{B_{G}}\left(L_{G}\right)$ on $G$ is multiplicative. Our interest in $B$-field transformations of multiplicative Dirac structures is motivated by the work carried out in [Bursztyn 2005; Bursztyn and Radko 2003], where the authors study the connection between certain $B$-field transformations of symplectic and Poisson groupoids and the notion of Morita equivalence of Poisson manifolds.

3B6. Generalized complex groupoids. Generalized complex structures were introduced in [Hitchin 2003] and further developed in [Gualtieri 2003]. Given a smooth manifold $M$, one can consider the complexified vector bundle $\mathbb{T}_{\mathbb{C}} M:=\mathbb{T} M \otimes \mathbb{C}$ endowed with the complex Courant bracket and the complex pairing $\langle\cdot, \cdot\rangle$. A generalized complex structure on $M$ is a complex Dirac structure $L \subseteq \mathbb{T}_{\mathbb{C}} M$ such that $L \cap \bar{L}=\{0\}$, where $\bar{L}$ denotes the conjugate of $L$. Complexified versions of multiplicative Dirac structures give rise to generalized complex groupoids. More concretely, let $G$ be a Lie groupoid equipped with a generalized complex structure $L_{G}$. We say that $\left(G, L_{G}\right)$ is a generalized complex groupoid if $L_{G} \subseteq \mathbb{T}_{\mathbb{C}} G$ is a Lie subgroupoid. Generalized complex groupoids were introduced in [Jotz et al. 2012] under the name of Glanon groupoids. Structures such as symplectic groupoids and holomorphic Poisson groupoids are special instances of generalized complex groupoids.

\section{Dirac algebroids}

In this section we study Lie algebroids equipped with Dirac structures compatible with both the linear and Lie algebroid structure.

4A. Definition and main examples. Let $A \rightarrow M$ be a vector bundle. A Poisson bivector $\pi_{A}$ on $A$ is linear if the map $\pi_{A}^{\sharp}: T^{*} A \rightarrow T A$ is a morphism of double vector bundles. Similarly, a 2-form $\omega_{A}$ on $A$ is linear if the map $\omega_{A}^{\sharp}: T A \rightarrow T^{*} A$ is a morphism of double vector bundles. The bundle map $\omega_{A}^{\sharp}$ in this case covers a bundle morphism $\lambda: T M \rightarrow A^{*}$. As shown in [Konieczna and Urbański 1999], a linear 2-form $\omega_{A}$ on a vector bundle $A \rightarrow M$ is closed if and only if $\omega_{A}=-\left(\lambda^{t}\right)^{*} \omega_{\text {can }}$, 
where $\omega_{\text {can }}$ is the canonical symplectic form on $T^{*} M$ and $\lambda^{t}: A \rightarrow T^{*} M$ is a fiberwise dual map of $\lambda: T M \rightarrow A^{*}$. The definition below includes both linear Poisson bivectors and linear closed 2-forms as special instances.

Definition 4.1. A Dirac structure $L_{A}$ on $A$ is called linear if $L_{A} \subseteq \mathbb{\mathbb { A }} A$ is a double vector subbundle of $\mathbb{T} A$.

A linear Dirac structure $L_{A} \subseteq \mathbb{T} A$ is not only a vector bundle over $A$, but also a vector bundle over a subbundle $E \subseteq T M \oplus A^{*}$. It follows directly from the definition that graphs of linear Poisson bivector and linear closed 2-forms define linear Dirac structures. Linear Dirac structures arise also in connection with Lagrangian and Hamiltonian mechanics, see for example, [Grabowska and Grabowski 2011].

Assume now that $A \rightarrow M$ carries also a Lie algebroid structure. Consider the direct sum Lie algebroid $\mathbb{T} A=T A \oplus T^{*} A$, whose base manifold is $T M \oplus A^{*}$.

Definition 4.2. A Dirac structure $L_{A}$ on $A$ is called morphic if $L_{A}$ is a linear Dirac structure that is also a Lie subalgebroid of $\mathbb{T} A$.

We denote by $\operatorname{Dir}_{\text {morph }}(A)$ the space of morphic Dirac structures on the Lie algebroid $A$.

A pair $\left(A, L_{A}\right)$, where $A$ is a Lie algebroid endowed with a morphic Dirac structure $L_{A}$, will be referred to as a Dirac algebroid.

Example 4.3. Let $\pi_{A}$ be a linear Poisson bivector on a Lie algebroid $A \rightarrow M$. Then, the Dirac structure given by the graph of $\pi_{A}$ is morphic if and only if $\pi_{A}^{\sharp}: T^{*} A \rightarrow T A$ is a Lie algebroid morphism. As shown in [Mackenzie and Xu 1994], this is equivalent to the pair $\left(A, A^{*}\right)$ being a Lie bialgebroid.

Example 4.4. Let $\omega_{A}$ be a linear closed 2-form on a Lie algebroid $A \rightarrow M$, that is, $\omega_{A}=-\sigma^{*} \omega_{c a n}$, for some bundle map $\sigma: A \rightarrow T^{*} M$. The Dirac structure defined by the graph of $\omega_{A}$ is morphic if and only if $\omega_{A}^{\sharp}: T A \rightarrow T^{*} A$ is a Lie algebroid morphism. Equivalently, as shown in [Bursztyn et al. 2009a], the bundle map $\sigma: A \rightarrow T^{*} M$ is an IM-2-form on $A$. The notion of IM-2-form was introduced in [Bursztyn et al. 2004] motivated by the problem of the integration of Dirac structures. See also [Arias Abad and Crainic 2011], where IM-2-forms arise in connection with the Weil algebra and the Van Est isomorphism.

4B. More examples of Dirac algebroids. In addition to both morphic Poisson structures and morphic closed 2-forms, there are more examples of morphic Dirac structures, which we proceed to explain below.

4B1. Foliated algebroids. Let $A$ be a Lie algebroid and $F_{A} \subseteq T A$ an involutive subbundle which is also a Lie subalgebroid of $T A \rightarrow T M$. In this case we say that $\left(A, F_{A}\right)$ is a foliated algebroid. One can easily check that the Dirac structure $F_{A} \oplus F_{A}^{\circ} \subseteq \mathbb{T} A$ is a morphic Dirac structure. Foliated algebroids were studied 
in [Hawkins 2008] as a way to promote the notion of polarization in geometric quantization to the category of Lie algebroids. Also, a detailed discussion about foliated algebroids can be found in [Jotz and Ortiz 2012].

4B2. Dirac Lie algebras. Let $\mathfrak{g}$ be a Lie algebra. In this case, morphic Dirac structures are Lie subalgebroids of $T \mathfrak{g} \oplus T^{*} \mathfrak{g} \rightarrow \mathfrak{g}^{*}$. It follows from [Ortiz 2008] that Dirac Lie algebras are suitable pull-backs of Lie bialgebras.

4B3. Tangent lifts of Dirac algebroids. Let $\left(A, L_{A}\right)$ be a Dirac algebroid. Consider the tangent Dirac structure $L_{T A}$ on $T A$. By definition, the tangent Dirac structure is given by $L_{T A}:=\left(J_{A} \oplus \Theta_{A}\right)\left(T L_{A}\right)$, where $T L_{A} \rightarrow T M$ is the tangent algebroid associated to the Dirac structure $L_{A}$ viewed as a Lie algebroid over $A$. Since the bundle map $J_{A} \oplus \Theta_{A}: T T A \oplus T T^{*} A \rightarrow T T A \oplus T^{*} T A$ is a Lie algebroid isomorphism, we conclude that $L_{T A} \subseteq \mathbb{T} T A$ is a Lie subalgebroid. Therefore, the pair $\left(T A, L_{T A}\right)$ is a Dirac algebroid.

4B4. Symmetries of Dirac algebroids. Let $\left(A, L_{A}\right)$ be a Dirac algebroid. Consider a Lie group $H$ acting on $A$ by Lie algebroid automorphisms. Assume that the action is free and proper and that the $H$-orbits coincide with the characteristic leaves of $L_{A}$. One can check that the orbit space $A / H$ inherits a Lie algebroid structure over $M / H$, making the quotient map $A \rightarrow A / H$ into a Lie algebroid morphism. Since the $H$-orbits are exactly the characteristic leaves of $L_{A}$, one concludes that $A / H$ is equipped with a unique Poisson bivector $\pi_{\text {red }}$ determined by the fact that $A \rightarrow A / H$ is a forward and backward Dirac map. Since $L_{A}$ is morphic, we conclude that $\pi_{\text {red }}$ is a morphic Poisson structure on $A / H$. In particular, due to [Mackenzie and Xu 1994], the pair $\left(A / H,(A / H)^{*}\right)$ is a Lie bialgebroid. In the special case where $L_{A}$ is the graph of a morphic Poisson structure on $A$ and the action is Hamiltonian in the sense of [Fernandes and Iglesias $\geq 2013$ ], this recovers the reduction of Lie bialgebroids carried out in [Fernandes and Iglesias $\geq 2013$ ].

4B5. Morphic B-field transformations. Let $\left(A, L_{A}\right)$ be a Dirac algebroid. Associated to a morphic closed 2-form $B_{A}$ on $A$ is the Lie algebroid automorphism $\tau_{B_{A}}: \mathbb{T} A \rightarrow \mathbb{T} A,(X, \alpha) \mapsto\left(X, \alpha+i_{X} B_{A}\right)$. The Dirac structure $\tau_{B_{A}}\left(L_{A}\right) \subseteq \mathbb{T} A$ obtained out of $L_{A}$ by applying the B-field transformation $\tau_{B_{A}}$ is morphic. Therefore, the pair $\left(A, \tau_{B_{A}}\right)$ is a Dirac algebroid. In particular, $B$-field transformations of morphic Poisson structures (that is, Lie bialgebroid structures on $\left(A, A^{*}\right)$ ) by morphic closed 2-forms are always morphic Dirac structures. If the $B$-field transformation is admissible, that is, the resulting Dirac structure is the graph of a Poisson bivector, such a bivector is necessarily morphic as well. In particular, we get a new bialgebroid structure on $\left(A, A^{*}\right)$ referred to as a gauge transformation of the Lie bialgebroid $\left(A, A^{*}\right)$. Gauge transformations of Lie bialgebroids were introduced in [Bursztyn 2005] motivated by the study of gauge transformations of Poisson groupoids and Morita equivalence of Poisson manifolds. 
4B6. Generalized complex algebroids. Let $A \rightarrow M$ be a Lie algebroid. Consider the complexified Lie algebroid $\mathbb{T}_{\mathbb{C}} A=\left(T A \oplus T^{*} A\right) \otimes \mathbb{C}$ whose base manifold is $\left(T M \oplus A^{*}\right) \otimes \mathbb{C}$. A generalized complex structure $L_{A}$ on $A$ is morphic if $L_{A} \subseteq \mathbb{T}_{\mathbb{C}} A$ is a Lie subalgebroid. In this case, we say that $\left(A, L_{A}\right)$ is a generalized complex algebroid. The notion of generalized complex algebroid was introduced in [Jotz et al. 2012] under the name of Glanon algebroids. Generalized complex algebroids include holomorphic Poisson structures and holomorphic Lie bialgebroids as particular cases.

\section{Infinitesimal description of multiplicative Dirac structures}

This section is the main part of the present work. Here we show that Dirac algebroids correspond to the infinitesimal counterpart of Dirac groupoids.

5A. The canonical $\mathscr{C A}$-groupoid. The main idea for studying multiplicative Dirac structures infinitesimally is based on the following observation. Given a Lie groupoid $G$ over $M$, the canonical geometric objects associated to $\mathbb{T} G$ that are used to define Dirac structures (symmetric pairing and Courant bracket) are suitably compatible with the groupoid structure of $\mathbb{T} G$. This compatibility makes $\mathbb{T} G$ into a $\mathscr{C} A$-groupoid. The notion of $\mathscr{C} \mathscr{A}$-groupoid was suggested by Mehta [2009] and further studied by Li-Bland and Ševera [2011]. More precisely, let $\langle\cdot, \cdot\rangle_{G}$ be the nondegenerate symmetric pairing on the direct sum Lie groupoid $\mathbb{T} G$.

Proposition 5.1. The canonical pairing defines a morphism of Lie groupoids

$$
\langle\cdot, \cdot\rangle_{G}: \mathbb{\mathbb { N }} \oplus \mathbb{\mathbb { V }} G \rightarrow \mathbb{R},
$$

where $\mathbb{R}$ is equipped with the usual abelian group structure.

Proof. Since $\mathbb{R}$ is a groupoid over a point, we only need to check the compatibility of $\langle\cdot, \cdot\rangle_{G}$ with the corresponding groupoid multiplications. For that, consider elements $\left(X_{g} \oplus \alpha_{g}\right),\left(Y_{g} \oplus \beta_{g}\right) \in \mathbb{T}_{g} G$ and $\left(X_{h}^{\prime} \oplus \alpha_{h}^{\prime}\right),\left(Y_{h}^{\prime} \oplus \beta_{h}^{\prime}\right) \in \mathbb{T}_{h} G$. Then by definition of the groupoid structure on $\mathbb{T} \oplus \oplus \mathbb{T} G$, we have

$$
\begin{aligned}
\left(\left(X_{g} \oplus \alpha_{g}\right) \oplus\left(Y_{g} \oplus \beta_{g}\right)\right) *\left(\left(X_{h}^{\prime} \oplus \alpha_{h}^{\prime}\right) \oplus\left(Y_{h}^{\prime} \oplus \beta_{h}^{\prime}\right)\right) \\
=\left(X_{g} \bullet X_{h}^{\prime} \oplus \alpha_{g} \circ \alpha_{h}^{\prime}\right) \oplus\left(Y_{g} \bullet Y_{h}^{\prime} \oplus \beta_{g} \circ \beta_{h}^{\prime}\right),
\end{aligned}
$$

therefore one gets

$$
\begin{aligned}
\left\langle\left(X_{g} \bullet X_{h}^{\prime} \oplus \alpha_{g} \circ \alpha_{h}^{\prime}\right),\left(Y_{g} \bullet Y_{h}^{\prime} \oplus \beta_{g} \circ \beta_{h}^{\prime}\right)\right\rangle_{G} & \\
& =\left(\alpha_{g} \circ \alpha_{h}^{\prime}\right)\left(Y_{g} \bullet Y_{h}^{\prime}\right)+\left(\beta_{g} \circ \beta_{h}^{\prime}\right)\left(X_{g} \bullet X_{h}^{\prime}\right) \\
& =\alpha_{g}\left(Y_{g}\right)+\alpha_{h}^{\prime}\left(Y_{h}^{\prime}\right)+\beta_{g}\left(X_{g}\right)+\beta_{h}^{\prime}\left(X_{h}^{\prime}\right) \\
& =\left\langle\left(X_{g} \oplus \alpha_{g}\right),\left(Y_{g}, \beta_{g}\right)\right\rangle_{G}+\left\langle\left(X_{h}^{\prime} \oplus \alpha_{h}^{\prime}\right),\left(Y_{h}^{\prime} \oplus \beta_{h}^{\prime}\right)\right\rangle_{G} .
\end{aligned}
$$


In order to explain the relation between the Courant bracket and the Lie groupoid structure on the direct sum vector bundle $\mathbb{T} G=T G \oplus T^{*} G$, we consider the direct product Courant algebroid $\mathbb{T} G \times \mathbb{T} G \rightarrow G \times G$. Every section $a^{(2)}$ of $\mathbb{T} G \times \mathbb{}$. can be written as

$$
a^{(2)}=a_{1} \circ p r_{1} \oplus a_{2} \circ p r_{2},
$$

where $a_{1}, a_{2}$ are sections of $\mathbb{T} G$, and $p r_{1}, p r_{2}: \mathbb{T} G \times \mathbb{T} G \rightarrow \mathbb{T} G$ denote the natural projections. The direct product bracket on sections of $\mathbb{T} G \times \mathbb{T} G$ is defined as usual;

$$
\left[a^{(2)}, \bar{a}^{(2)}\right]=\llbracket a_{1}, \bar{a}_{1} \rrbracket \circ p r_{1} \oplus \llbracket a_{2}, \bar{a}_{2} \rrbracket \circ p r_{2},
$$

and the anchor map $\rho_{(\mathbb{T} G)_{(2)}}: \mathbb{T} G \times \mathbb{T} G \rightarrow T G \times T G$ is given by the canonical componentwise projection.

Proposition 5.2. Let $m_{\mathbb{T}}:(\mathbb{T} G)_{(2)} \rightarrow \mathbb{} G$ denote the groupoid multiplication of $\mathbb{} \mathbb{} G=T G \oplus T^{*} G$. If $a, b, a_{i}, b_{i} \in \Gamma(\mathbb{T} G), i=1,2$ are sections such that

$$
m_{\mathbb{T}} \circ\left(a_{1}, a_{2}\right)=a \circ m_{G}, \quad m_{\mathbb{T}} \circ\left(b_{1}, b_{2}\right)=b \circ m_{G},
$$

then the following identities hold:

(i) $\operatorname{Tm}_{G}\left(\rho_{(\mathbb{T} G)_{(2)}}\left(X_{g}^{1} \oplus \alpha_{g}^{1}, X_{h}^{2} \oplus \alpha_{h}^{2}\right)\right)=X_{g}^{1} \bullet X_{h}^{2}$;

(ii) $m_{\rrbracket} \circ\left(\llbracket a_{1}, b_{1} \rrbracket, \llbracket a_{2}, b_{2} \rrbracket\right)=\llbracket a, b \rrbracket \circ m_{G}$.

Proof. We begin by checking (i). For that, consider a section $a^{(2)}=a_{1} \circ p r_{1} \oplus a_{2} \circ p r_{2}$ of $(\mathbb{T} G)_{(2)}$, where $a_{1}=X^{1} \oplus \alpha^{1}$ and $a_{2}=X^{2} \oplus \alpha^{2}$ are sections of $\mathbb{T} G$. The multiplication on the Lie groupoid $\mathbb{T} G$ maps the section $a^{(2)}$ into

$$
m_{\mathbb{T}}\left(a_{1} \circ p r_{1} \oplus a_{2} \circ p r_{2}\right)(g, h)=X_{g}^{1} \bullet X_{h}^{2} \oplus \alpha_{g}^{1} \circ \alpha_{h}^{2} .
$$

Applying the anchor map of $\mathbb{T} G$ we obtain

$$
\rho_{\mathbb{}}\left(X_{g}^{1} \bullet X_{h}^{2} \oplus \alpha_{g}^{1} \circ \alpha_{h}^{2}\right)=X_{g}^{1} \bullet X_{h}^{2} .
$$

On the other hand, the componentwise anchor map of $(\mathbb{T} G)_{(2)}$ applied to the section $a^{(2)}$ gives rise to

$$
\rho_{(\mathbb{T} G)_{(2)}}\left(a_{1} \circ p r_{1} \oplus a_{2} \circ p r_{2}\right)(g, h)=\left(X_{g}^{1}, X_{h}^{2}\right),
$$

which followed by the derivative of $m_{G}: G_{(2)} \rightarrow G$ yields

$$
\operatorname{Tm}_{G}\left(\rho_{(\mathbb{T} G)_{(2)}}\left(X_{g}^{1} \oplus \alpha_{g}^{1}, X_{h}^{2} \oplus \alpha_{h}^{2}\right)\right)=X_{g}^{1} \bullet X_{h}^{2},
$$

as required. In order to prove identity (ii), one considers

$$
\begin{aligned}
& m_{\mathbb{}} \circ a^{(2)}=a \circ m_{G}, \\
& m_{\mathbb{}} \circ \bar{a}^{(2)}=\bar{a} \circ m_{G},
\end{aligned}
$$


where $a^{(2)}, \bar{a}^{(2)} \in \Gamma_{G_{(2)}}\left((\mathbb{T} G)_{(2)}\right)$ and $a, \bar{a} \in \Gamma_{G}(\mathbb{T} G)$. More concretely, write down sections as

$$
\begin{aligned}
a^{(2)} & =\left(X^{1} \oplus \alpha^{1}\right) \circ p r_{1} \oplus\left(X^{2} \oplus \alpha^{2}\right) \circ p r_{2}, \\
\bar{a}^{(2)} & =\left(\bar{X}^{1} \oplus \bar{\alpha}^{1}\right) \circ p r_{1} \oplus\left(\bar{X}^{2} \oplus \bar{\alpha}^{2}\right) \circ p r_{2}, \\
a & =Y \oplus \beta, \\
\bar{a} & =\bar{Y} \oplus \bar{\beta} .
\end{aligned}
$$

The identities (9), (10) then become

$$
\begin{aligned}
& X_{g}^{1} \bullet X_{h}^{2} \oplus \alpha_{g}^{1} \circ \alpha_{h}^{2}=Y_{g h} \oplus \beta_{g h}, \\
& \bar{X}_{g}^{1} \bullet \bar{X}_{h}^{2} \oplus \bar{\alpha}_{g}^{1} \circ \bar{\alpha}_{h}^{2}=\bar{Y}_{g h} \oplus \bar{\beta}_{g h},
\end{aligned}
$$

for any composable pair $(g, h) \in G \times G$. Now it follows directly from the definition of the direct product bracket that

$$
\begin{aligned}
& {\left[a^{(2)}, \bar{a}^{(2)}\right]} \\
& \quad=\left(\left[X^{1}, \bar{X}^{1}\right] \oplus \mathscr{L}_{X^{1}} \bar{\alpha}^{1}-i_{\bar{X}^{1}} d \alpha^{1}\right) \circ p r_{1} \oplus\left(\left[X^{2}, \bar{X}^{2}\right] \oplus \mathscr{L}_{X^{2}} \bar{\alpha}^{2}-i_{\bar{X}^{2}} d \alpha^{2}\right) \circ p r_{2} .
\end{aligned}
$$

Then, composing with the groupoid multiplication of $\mathbb{T} G$, we have

$$
\begin{aligned}
m_{\mathbb{T}} \circ\left[a^{(2)}, \bar{a}^{(2)}\right]_{(g, h)} & \\
& =\left[X^{1}, \bar{X}^{1}\right]_{g} \bullet\left[X^{2}, \bar{X}^{2}\right]_{h} \oplus\left(\mathscr{L}_{X^{1}} \bar{\alpha}^{1}-i_{\bar{X}^{1}} d \alpha^{1}\right)_{g} \circ\left(\mathscr{L}_{X^{2}} \bar{\alpha}^{2}-i_{\bar{X}^{2}} d \alpha^{2}\right)_{h} .
\end{aligned}
$$

On the other hand,

$$
\llbracket a, \bar{a} \rrbracket \circ m_{G}(g, h)=[Y, \bar{Y}]_{g h} \oplus\left(\mathscr{L}_{Y} \bar{\beta}-i_{\bar{Y}} d \beta\right)_{g h},
$$

and using the identities (11) and (12) one concludes that

$$
[Y, \bar{Y}]_{g h}=\left[X^{1}, \bar{X}^{1}\right]_{g} \bullet\left[X^{2}, \bar{X}^{2}\right]_{h} .
$$

Thus, the tangent component of $\llbracket a, \bar{a} \rrbracket_{g h}$ coincides with the tangent component of $m_{\mathbb{V}} \circ\left[a^{(2)}, \bar{a}^{(2)}\right]_{(g, h)}$. It remains to show that we also have the equality of the corresponding cotangent parts. This is equivalent to showing that

$$
\begin{aligned}
& \left(\mathscr{L}_{Y} \bar{\beta}-\mathscr{L}_{\bar{Y}} \beta-d\langle\beta, \bar{Y}\rangle\right)_{g h} \\
& \quad=\left(\mathscr{L}_{X^{1}} \bar{\alpha}^{1}-\mathscr{L}_{\bar{X}^{1}} \alpha^{1}-d\left\langle\alpha^{1}, \bar{X}^{1}\right\rangle\right)_{g} \circ\left(\mathscr{L}_{X^{2}} \bar{\alpha}^{2}-\mathscr{L}_{\bar{X}^{2}} \alpha^{2}-d\left\langle\alpha^{2}, \bar{X}^{2}\right\rangle\right)_{h},
\end{aligned}
$$

for every composable pair $(g, h) \in G_{(2)}$. In order to prove this identity, we need to check that the left hand side $(L H S)$, and the right hand side $(R H S)$ above coincide at elements of the form $U_{g} \bullet V_{h}$. For that consider the 1-form on $G$ defined by $\gamma:=\mathscr{L}_{Y} \bar{\beta}-\mathscr{L}_{\bar{Y}} \beta-d\langle\beta, \bar{Y}\rangle$. We can look at the pull-back 1-form $m_{G}^{*} \gamma \in \Omega^{1}\left(G_{(2)}\right)$, which at every tangent vector $\left(U_{g}, V_{h}\right) \in T_{(g, h)} G_{(2)}$ is given by

$$
\left(m_{G}^{*} \gamma\right)_{(g, h)}\left(U_{g}, V_{h}\right)=\gamma_{g h}\left(U_{g} \bullet V_{h}\right)=(L H S)\left(U_{g} \bullet V_{h}\right) .
$$


The pull-back form $m_{G}^{*} \gamma$ involves three terms. Let us analyze the first term $m_{G}^{*}\left(\mathscr{L}_{Y} \bar{\beta}\right)$ of this pull-back form. It follows from the relation $Y=\left(m_{G}\right)_{*}\left(X^{1}, X^{2}\right)$ that

$$
m_{G}^{*}\left(\mathscr{L}_{Y} \bar{\beta}\right)=\mathscr{L}_{\left(X^{1}, X^{2}\right)} m_{G}^{*} \bar{\beta} .
$$

Notice that (12) implies that

$$
\begin{aligned}
\left(m_{G}^{*} \bar{\beta}\right)_{(g, h)}\left(U_{g}, V_{H}\right) & =\bar{\beta}_{g h}\left(U_{g} \bullet V_{h}\right)=\left(\bar{\alpha}_{g}^{1} \circ \bar{\alpha}_{h}^{2}\right)\left(U_{g} \bullet V_{h}\right) \\
& =\bar{\alpha}_{g}^{1}\left(U_{g}\right)+\bar{\alpha}_{h}^{2}\left(V_{h}\right)=\left(\bar{\alpha}^{1}, \bar{\alpha}^{2}\right)_{(g, h)}\left(U_{g}, V_{h}\right) .
\end{aligned}
$$

That is, $m_{G}^{*}\left(\mathscr{L}_{Y} \bar{\beta}\right)=\mathscr{L}_{X^{1}} \bar{\alpha}^{1} \oplus \mathscr{L}_{X^{2}} \bar{\alpha}^{2}$. A similar argument can be applied to the other terms of the pull-back form $m_{G}^{*} \gamma$, yielding

$$
\begin{aligned}
(L H S)\left(U_{g} \bullet V_{h}\right)= & \left(m_{G}^{*} \gamma\right)_{(g, h)}\left(U_{g}, V_{h}\right) \\
= & \left(\mathscr{L}_{X^{1}} \bar{\alpha}^{1}\right)_{g}\left(U_{g}\right)+\left(\mathscr{L}_{X^{2}} \bar{\alpha}^{2}\right)_{h}\left(V_{h}\right)-\left(\mathscr{L}_{\bar{X}^{1}} \alpha^{1}\right)_{g}\left(U_{g}\right) \\
& -\left(\mathscr{L}_{\bar{X}^{2}} \alpha^{2}\right)_{h}\left(V_{h}\right)-d\left\langle\alpha^{1}, \bar{X}^{1}\right\rangle_{g}\left(U_{g}\right)-d\left\langle\alpha^{2}, \bar{X}^{2}\right\rangle_{h}\left(V_{h}\right) \\
= & (R H S)\left(U_{g} \bullet V_{h}\right) .
\end{aligned}
$$

Thus RHS and LHS coincide at elements of the form $U_{g} \bullet V_{h}$, and we conclude that $\left(m_{\mathbb{T}}, m_{G}\right)$ is bracket preserving.

Recall that, given a Courant algebroid $(\mathbb{E}, \rho, \mathbb{[} \cdot, \cdot \rrbracket)$ over smooth manifold $M$ and a submanifold $Q \subseteq M$, a Dirac structure supported on $Q$ (see [Alekseev and Xu 2011; Bursztyn et al. 2009b]) is a subbundle $\left.K \subset \mathbb{E}\right|_{Q}$ such that $K_{x} \subseteq \mathbb{E}_{x}$ is Lagrangian for all $x \in Q$ and the following conditions are fulfilled:

(1) $\rho(K) \subseteq T Q$;

(2) whenever $a_{1}, a_{2} \in \Gamma(\mathbb{E})$ satisfy $\left.a_{1}\right|_{Q},\left.a_{2}\right|_{Q} \in \Gamma(K)$, then $\llbracket a_{1},\left.a_{2} \rrbracket\right|_{Q} \in \Gamma(K)$.

Dirac structures with support were used in [Bursztyn et al. 2009b] to introduce a natural notion of morphism between Courant algebroids. Let $\mathbb{E}_{1}, \mathbb{E}_{2}$ be Courant algebroids over $M, N$, respectively. A Courant algebroid morphism from $\mathbb{E}_{1}$ to $\mathbb{E}_{2}$ is a Dirac structure in $\mathbb{E}_{2} \times \overline{\mathbb{E}}_{1}$ supported on $\operatorname{Graph}(f)$, where $f: M \rightarrow N$ is a smooth map. Here $\mathbb{E}_{1}$ denotes the Courant algebroid structure on the vector bundle $\mathbb{E}_{1}$ with the same bracket on $\Gamma\left(\mathbb{E}_{1}\right)$, anchor map and minus the usual symmetric pairing.

Combining Propositions 5.1 and 5.2, we obtain:

Proposition 5.3. Let $G$ be a Lie groupoid over $M$ with multiplication map $m_{G}$ : $G_{(2)} \rightarrow G$. Let $m_{\mathbb{}}:(\mathbb{T} G)_{(2)} \rightarrow \mathbb{} G$ denote the groupoid multiplication on $\mathbb{T} G$. Then $\operatorname{Graph}\left(m_{\mathbb{T}}\right) \subseteq \mathbb{T} G \times \overline{\mathbb{T} G \times \mathbb{T} G}$ is a Dirac structure supported on $\operatorname{Graph}\left(m_{G}\right) \subseteq$ $G \times G \times G$. That is, $\operatorname{Graph}\left(m_{\mathbb{T}}\right)$ is a Courant algebroid morphism from $\mathbb{T} G \times \mathbb{T} G$

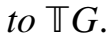


Using the terminology of [Li-Bland and Ševera 2011], Proposition 5.3 says that $\mathbb{G}$ with its canonical Courant algebroid structure and groupoid multiplication is an example of $\mathscr{C} \mathscr{A}$-groupoid. See also Example 2.3.1 of [Li-bland 2012].

\section{B. The $\mathscr{L} A$-groupoid of a multiplicative Dirac structure.}

5B1. Review of $\mathscr{L} \mathscr{A}$-groupoids. An $\mathscr{L} \mathscr{A}$-groupoid is a Lie groupoid object in the category of Lie algebroids. More precisely, an LLA-groupoid [Mackenzie 1992] is a square

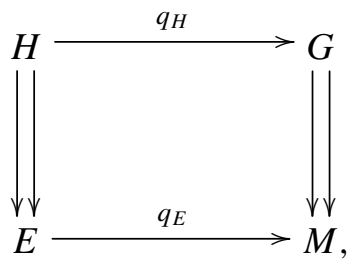

where the single arrows denote Lie algebroids and the double arrows denote Lie groupoids. These structures are compatible in the sense that all the structure mappings (that is, source, target, unit section, inversion and multiplication) defining the Lie groupoid $H$ are Lie algebroid morphisms over the corresponding structure mappings which define the Lie groupoid $G$. We also require that the anchor map $\rho_{H}: H \rightarrow T G$ be a groupoid morphism over the anchor map $\rho_{E}: E \rightarrow T M$. Here $T G$ is endowed with the tangent groupoid structure over $T M$. For describing the square given by an $\mathscr{L} \mathscr{A}$-groupoid we use the notation $(H, G, E, M)$. It is worthwhile to explain how the groupoid multiplication defines a morphism of Lie algebroids. For that, let $m_{H}: H_{(2)} \subseteq H \times H \rightarrow H$ denote the groupoid multiplication of $H$, and similarly let $m_{G}: G_{(2)} \subseteq G \times G \rightarrow G$ denote the multiplication of $G$. The direct product vector bundle $H \times H \rightarrow G \times G$ inherits a natural Lie algebroid structure, and we have a Lie subalgebroid $H_{(2)}$ over $G_{(2)}$ which is just a pull-back algebroid, see for example, [Higgins and Mackenzie 1990] for details about the pull-back operation in the category of Lie algebroids. With respect to this Lie algebroid structure, the multiplication map $m_{H}$ is required to be a Lie algebroid morphism covering $m_{G}$.

The Lie functor applied to an $\mathscr{L} \mathscr{A}$-groupoid (13) determines a double vector bundle

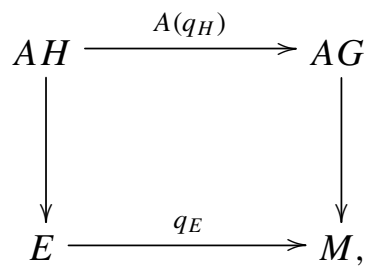

where each of the arrows define Lie algebroids. The top Lie algebroid structure 
is nontrivial, and it deserves a detailed explanation. The Lie algebroid structure $A H \rightarrow A G$ was constructed in [Mackenzie 2000] as a prolongation procedure similar to the tangent prolongation of a Lie algebroid, except that we replace the tangent functor by the Lie functor.

Definition 5.4. The prolonged anchor map $A H \rightarrow T(A G)$ is defined by

$$
\tilde{\rho}:=j_{G}^{-1} \circ A\left(\rho_{H}\right),
$$

where $j_{G}: T(A G) \rightarrow A(T G)$ is the canonical identification defined in Equation (5).

Now we study the space of sections $\Gamma_{A G}(A H)$.

Definition 5.5. A section $u \in \Gamma_{G}(H)$ is called a star section if there exists a section $u_{0} \in \Gamma_{M}(E)$ such that

(1) $\epsilon_{E} \circ u_{0}=u \circ \epsilon_{M}$,

(2) $s_{H} \circ u=u_{0} \circ s_{G}$.

Notice that since every star section $u: G \rightarrow H$ preserves the units and the source fibrations, we are allowed to apply the Lie functor to $u$, yielding a section $A(u)$ of the vector bundle $A H \stackrel{A\left(q_{H}\right)}{\longrightarrow} A G$.

Definition 5.6. Let $(H, G, E, M)$ be an $\mathscr{L} A$-groupoid. The core of $H$ is the vector bundle over $M$ defined by

$$
K:=\epsilon_{M}^{*} \operatorname{ker}\left(s_{H}\right) .
$$

Every section $k \in \Gamma(K)$ induces a section $k_{H} \in \Gamma_{G}(H)$ in the following way:

$$
k_{H}(g):=k\left(t_{G}(g)\right) 0_{g}^{H},
$$

where $0_{g}^{H}$ is the zero element in the fiber $H_{g}$ above $g \in G$. Notice that for every section $k \in \Gamma(K)$ the induced section $k_{H} \in \Gamma_{G}(H)$ satisfies

$$
k_{H} \circ \epsilon_{M}=k \text {. }
$$

It was proved in [Mackenzie 2000] that the core of the double vector bundle $(A H, A G, E, M)$ is the vector bundle $K \rightarrow M$. Notice that a core element $k \in K$ induces a Lie algebroid element $\bar{k} \in A H$. Indeed, we observe that every element in $A H$ has the form

$$
W=\left.\frac{d}{d t}\left(h_{t}\right)\right|_{t=0},
$$

where $h_{t}$ is a curve in $H$ sitting in a fixed source fiber $s_{H}^{-1}(e)$ with $h_{0}=\epsilon_{E}(e)$. Thus, for every core element $k \in K$ above $x \in M$ - that is, $s_{H}(k)=0_{x}^{E}$ and $q_{H}(k)=\epsilon_{M}(x)$ - there exists a natural element $\bar{k} \in A H$, defined by

$$
\bar{k}:=\left.\frac{d}{d t}(t k)\right|_{t=0} \text {. }
$$


Definition 5.7. Given a section $k \in \Gamma(K)$, the core section induced by $k$ is the section $k^{\text {core }} \in \Gamma_{A G}(A H)$ defined by

$$
k^{\text {core }}\left(u_{x}\right):=A\left(0^{H}\right) u_{x}+\overline{k(x)} .
$$

Proposition 5.8 [Mackenzie 2000]. The space of sections $\Gamma_{A G}(A H)$ is generated by sections of the form $A(u)$, where $u: G \rightarrow H$ is a star section, and by sections of the form $k^{\text {core }}$, where $k: M \rightarrow K$ is a section of the core of $H$.

The Lie bracket on $\Gamma_{A G}(A H)$ is defined in terms of star sections and core sections. First we observe that whenever $u, v \in \Gamma_{G}(H)$ are star sections, then the Lie bracket $[u, v] \in \Gamma_{G}(H)$ is also a star section. Thus the Lie bracket between sections of the form $A(u), A(v)$ is defined by

$$
[A(u), A(v)]=A([u, v]) .
$$

The bracket of a pair of core sections is defined by

$$
\left[k_{1}^{\text {core }}, k_{2}^{\text {core }}\right]=0 .
$$

In order to define the bracket of a star section and a core section we notice that every star section $u: G \rightarrow H$ induces a covariant differential operator

$$
D_{u}: \Gamma(K) \rightarrow \Gamma(K), \quad k \mapsto\left[u, k_{H}\right] \circ \epsilon_{M} .
$$

Now we define $\left[A(u), k^{\text {core }}\right]=\left(D_{u}(k)\right)^{\text {core }}$.

The Lie bracket of other sections of $\Gamma_{A G}(A H)$ is defined by requiring the Leibniz rule

$$
\left[w, f w^{\prime}\right]=f\left[w, w^{\prime}\right]+\left(\mathscr{L}_{\tilde{\rho}(w)} f\right) w^{\prime} .
$$

The vector bundle $A H \stackrel{A\left(q_{H}\right)}{\longrightarrow} A G$ endowed with the anchor map

$$
\tilde{\rho}=j_{G}^{-1} \circ A(\rho)
$$

and the Lie bracket $[\cdot, \cdot]$ on $\Gamma_{A G}(A H)$ becomes a Lie algebroid called the prolonged Lie algebroid induced by $H \rightarrow G$, see [Mackenzie 2000].

Although the following remark is not mentioned in [Mackenzie 2000], it is important to notice that Mackenzie's construction of the prolonged Lie algebroid is natural in the following sense.

Proposition 5.9. Let $(H, G, E, M)$ be an $\mathscr{L} A$-groupoid. Consider the canonical embeddings $i_{A H}: A H \rightarrow T H$ and $i_{A G}: A G \rightarrow T G$. Endow $T H \rightarrow T G$ with the tangent algebroid structure and $A H \rightarrow A G$ with the prolonged algebroid structure. Then $i_{A H}$ is a Lie algebroid morphism covering $i_{A G}$. 
Recall that (see for example, [Mackenzie 2005]) a vector bundle map $\Psi: A \rightarrow B$, covering $\psi: M \rightarrow N$, is a Lie algebroid morphism if

$$
\rho_{B} \circ \Psi=T \psi \circ \rho_{A},
$$

and the following compatibility with brackets holds: For sections $u, v \in \Gamma(A)$ such that $\Psi(u)=\sum_{j} f_{j} \psi^{*} u_{j}$ and $\Psi(v)=\sum_{i} g_{i} \psi^{*} v_{i}$, where $f_{j}, g_{i} \in C^{\infty}(M)$ and $u_{j}, v_{i} \in \Gamma(B)$, we have

$$
\Psi\left([u, v]_{A}\right)=\sum_{i, j} f_{j} g_{i} \psi^{*}\left[u_{j}, v_{i}\right]_{B}+\sum_{i} \mathscr{L}_{\rho_{A}(u)} g_{i} \psi^{*} v_{i}-\sum_{j} \mathscr{L}_{\rho_{A}(v)} f_{j} \psi^{*} u_{j} .
$$

Proof. The compatibility with the anchor maps reads

$$
\rho_{T H} \circ i_{A H}=T i_{A G} \circ \tilde{\rho},
$$

which is exactly the definition of the prolonged anchor map.

Let us check now the compatibility with the Lie brackets. For that, consider a star section $u: G \rightarrow H$. Then, there are sections $T u: T G \rightarrow T H$ and $A(u): A G \rightarrow A H$. Both are related by $A(u)=\left.T u\right|_{A G}$. In particular, $i_{A H} \circ A(u)=T u \circ i_{A G}$ holds. Similarly, every section $k \in \Gamma(K)$ of the core of $H$ induces a section of the tangent prolongation $T H \rightarrow T G$. Indeed, first consider the induced section $k_{H} \in \Gamma_{G}(H)$ and then construct the core section $\hat{k}_{H} \in \Gamma_{T G}(T H)$ defined in the usual way:

$$
\hat{k}_{H}\left(X_{g}\right)=T\left(0^{H}\right) X_{g}+\overline{k_{H}(g)} .
$$

For every $x \in \epsilon_{M}(M) \subseteq G$ one has $k_{H}(x)=k(x)$, and thus at any $u_{x} \in(A G)_{x} \subseteq T_{x} G$ we get

$$
\hat{k}_{H}\left(u_{x}\right)=A\left(0^{H}\right) u_{x}+\overline{k(x)} .
$$

Hence we conclude that $i_{A H} \circ k^{\text {core }}=\hat{k}_{H} \circ i_{A G}$. Let us show that (15) holds for a pair of sections $A(u), A(v)$, where $u, v: G \rightarrow H$ are star sections. Indeed,

$$
i_{A H} \circ[A(u), A(v)]=i_{A H} \circ A[u, v]=T[u, v] \circ i_{A G}=[T u, T v] \circ i_{A G},
$$

as desired. It remains to show the bracket condition (15) for sections of the form $A(u), k^{\text {core }}$, where $u: G \rightarrow H$ is a star section and $k: M \rightarrow K$ is a section of the core. On the one hand, one has that

$$
i_{A H} \circ\left[A(u), k^{\mathrm{core}}\right]=i_{A H} \circ\left(D_{u} k\right)^{\mathrm{core}}=\left(\widehat{D_{u} k}\right)_{H} \circ i_{A G} .
$$

On the other hand,

$$
\left.\left[T u, \hat{k}_{H}\right] \circ i_{A G}=\widehat{\left[u, k_{H}\right.}\right] \circ i_{A G} .
$$

Notice that to conclude that (15) holds in this case it suffices to show that $\left.\left(\widehat{D_{u} k}\right)_{H} \circ i_{A G}=\widehat{\left[u, k_{H}\right.}\right] \circ i_{A G}$. Indeed, using the fact that $k=k_{H} \circ \epsilon_{M}$ for every 
section $k: M \rightarrow K$, we conclude that if $v_{x} \in A_{x} G$, then

$$
\begin{aligned}
\left.\widehat{\left[u, k_{H}\right.}\right]\left(u_{x}\right) & =T 0_{G}^{H}\left(u_{x}\right)+\left.\frac{d}{d t}\left(t\left[u, k_{H}\right](x)\right)\right|_{t=0} \\
& =T 0_{G}^{H}\left(u_{x}\right)+\left.\frac{d}{d t}\left(t\left(D_{u} k\right)_{H}(x)\right)\right|_{t=0}=\left(\widehat{\left.D_{u} k\right)_{H}}\left(v_{x}\right) .\right.
\end{aligned}
$$

5B2. Dirac groupoids as $\mathscr{L} \mathscr{A}$-groupoids. Let $L_{G}$ be a multiplicative Dirac structure on a Lie groupoid $G \rightrightarrows M$. This means that we have a $\mathscr{V} \mathscr{B}$-subgroupoid $L_{G} \rightrightarrows E$ of $\mathbb{T} G \rightrightarrows T M \oplus A^{*} G$, such that $L_{G} \subseteq \mathbb{T} G$ is also a Dirac subbundle. In particular there is a canonical Lie algebroid structure on $L_{G} \rightarrow G$ with anchor map $L_{G} \rightarrow T G$ the natural projection and Lie bracket $\llbracket \cdot, \cdot \rrbracket$ on $\Gamma_{G}\left(L_{G}\right)$. Given sections $e_{1}, e_{2}$ of $E$, there exist star sections $a_{1}, a_{2}$ of $L_{G}$ covering $e_{1}$ and $e_{2}$, respectively. Since $L_{G}$ is involutive with respect to the Courant bracket, we conclude that $\llbracket a_{1}, a_{2} \rrbracket$ is a star section of $L_{G}$ covering a section $e$ of $E$. We define $\left[e_{1}, e_{2}\right]:=e$. A straightforward computation shows that with respect to this Lie bracket and the natural projection $E \rightarrow T M$, the vector bundle $E \rightarrow M$ becomes a Lie algebroid.

Proposition 5.10. A multiplicative Dirac structure $L_{G}$ on $G$ gives rise to an $\mathscr{L} A$ groupoid

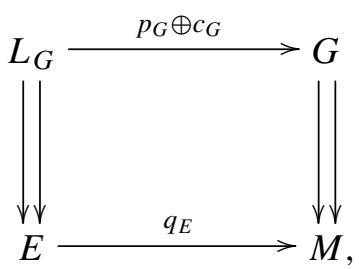

where $p_{G}$ and $c_{G}$ denote the tangent projection and the cotangent projection, respectively.

Proof. Since the structure mappings defining the Lie groupoid $L_{G} \rightrightarrows E$ are restrictions of the structure mappings of the tangent and cotangent groupoids, a straightforward computation shows that these structure mappings are Lie algebroid morphisms over the structure mapping of $G$. The fact that the multiplication on $L_{G}$ is a Lie algebroid morphism over the multiplication on $G$ follows from Proposition 5.2. An argument similar to the one used in the proof of Proposition 5.2 shows that the inversion map on $L_{G}$ is a Lie algebroid morphism.

5C. The Lie algebroid of a multiplicative Dirac structure. We let $G$ be a Lie groupoid over $M$ with Lie algebroid $A G$. Let $L_{G}$ be a multiplicative Dirac structure on a Lie groupoid $G$. According to Proposition 5.1, the canonical pairing $\langle\cdot, \cdot\rangle_{G}$ : $\mathbb{T} G \oplus \mathbb{T} G \rightarrow \mathbb{R}$ is a Lie groupoid morphism. Applying the Lie functor yields a nondegenerate symmetric pairing

$$
A\left(\langle\cdot, \cdot\rangle_{G}\right):\left(A(T G) \oplus A\left(T^{*} G\right)\right) \times_{A G}\left(A(T G) \oplus A\left(T^{*} G\right)\right) \rightarrow \mathbb{R} .
$$


Let $\langle\cdot, \cdot\rangle_{A G}$ denote the canonical nondegenerate symmetric pairing on $\mathbb{T}(A G)$. Recall that there exist canonical isomorphisms of Lie algebroids $j_{G}: T(A G) \rightarrow$ $A(T G)$ and $j_{G}^{\prime}: A\left(T^{*} G\right) \rightarrow T^{*}(A G)$ (see (5) and (6)). Since $\langle\cdot, \cdot\rangle_{A G}$ is just a suitable restriction of $T\langle\cdot, \cdot\rangle_{G}$, one concludes that the canonical map

$$
j_{G}^{-1} \oplus j_{G}^{\prime}: A(T G) \oplus A\left(T^{*} G\right) \rightarrow T(A G) \oplus T^{*}(A G)
$$

is a fiberwise isometry with respect to $A\left(\langle\cdot, \cdot\rangle_{G}\right)$ and $\langle\cdot, \cdot\rangle_{A G}$. This is a useful tool for transporting Lagrangian subbundles of $T G \oplus T^{*} G$ to Lagrangian subbundles of $T(A G) \oplus T^{*}(A G)$. For instance, given a $\mathscr{V} \mathscr{B}$-subgroupoid $L_{G}$ of $T G \oplus T^{*} G$, we can apply the Lie functor to obtain a $\mathscr{V} \mathscr{B}$-subalgebroid $A\left(L_{G}\right) \subseteq A(T G) \oplus A\left(T^{*} G\right)$. We mimic the construction of tangent Dirac structures, giving rise to a $\mathscr{V} \mathscr{B}$-subalgebroid of $T(A G) \oplus T^{*}(A G)$ defined by

$$
L_{A G}:=\left(j_{G}^{-1} \oplus j_{G}^{\prime}\right)\left(A\left(L_{G}\right)\right) .
$$

The following result is a straightforward consequence of the previous discussion.

Proposition 5.11. Let $L_{G} \subseteq T G \oplus T^{*} G$ be a $\mathscr{V} \mathscr{B}$-subgroupoid. Consider the associated $\mathscr{V} \mathscr{B}$-subalgebroid $L_{A G} \subseteq T(A G) \oplus T^{*}(A G)$. Then $L_{G}$ is isotropic with respect to $\langle\cdot, \cdot\rangle_{G}$ if and only if $L_{A G}$ is isotropic with respect to $\langle\cdot, \cdot\rangle_{A G}$.

In particular, if $L_{G} \subseteq T G \oplus T^{*} G$ is a $\mathscr{V} \mathscr{B}$-subgroupoid with associated $\mathscr{V} \mathscr{B}$ subalgebroid $L_{A G} \subseteq T(A G) \oplus T^{*}(A G)$ then $L_{G}$ is an almost Dirac structure on $G$ if and only if $L_{A G}$ is an almost Dirac structure on $A G$.

Now we want to deal with integrability issues. For that, consider a multiplicative Dirac structure $L_{G} \subseteq \mathbb{T} G$ and let $\left(L_{G}, G, E, M\right)$ be the associated $\mathscr{L} \mathscr{A}$-groupoid. Applying the Lie functor we obtain the prolonged Lie algebroid structure on $A\left(L_{G}\right) \rightarrow A G$, and we use the canonical map

$$
j_{G}^{-1} \oplus j_{G}^{\prime}: A(T G) \oplus A\left(T^{*} G\right) \rightarrow T(A G) \oplus T^{*}(A G)
$$

to define a Lie algebroid $L_{A G}=\left(j_{G}^{-1} \oplus j_{G}^{\prime}\right)\left(A\left(L_{G}\right)\right)$ over $A G$, characterized by the fact that $j_{G}^{-1} \oplus j_{G}^{\prime}: A\left(L_{G}\right) \rightarrow L_{A G}$ is a Lie algebroid isomorphism. We have seen that $L_{A G} \subseteq \mathbb{T}(A G)$ is a Lagrangian subbundle with respect to the canonical pairing $\langle\cdot, \cdot\rangle_{A G}$ on $\mathbb{T}(A G)$. We claim that the Lie bracket on $\Gamma_{A G}\left(L_{A G}\right)$ induced by the prolonged Lie bracket on $\Gamma_{A G}\left(A\left(L_{G}\right)\right)$ coincides with the Courant bracket. Indeed, since the tangent Lie algebroid $T L_{G} \rightarrow T G$ is isomorphic to $L_{T G} \rightarrow T G$, where the latter is equipped with the algebroid structure induced by the tangent Dirac structure $L_{T G} \subset T T G \oplus T^{*} T G$, and $A\left(L_{G}\right)$ is a Lie subalgebroid of $T L_{G}$ (Proposition 5.9), then the bracket on sections of $L_{A G}$ induced by the identification $A\left(L_{G}\right)=L_{A G}$ is exactly the restriction of the Courant bracket on $\Gamma\left(T(A G) \oplus T^{*}(A G)\right)$. We have proved: 
Theorem 5.12. Given a Lie groupoid $G$ with Lie algebroid $A G$, there is a canonical map

$$
\operatorname{Dir}_{\text {mult }}(G) \rightarrow \operatorname{Dir}_{\text {morph }}(A G), L_{G} \mapsto L_{A G}:=\left(j_{G}^{-1} \oplus j_{G}^{\prime}\right)\left(A\left(L_{G}\right)\right) .
$$

That is, up to a canonical identification, the Lie algebroid of a multiplicative Dirac structure $L_{G} \subset \mathbb{T} G$ defines a Dirac structure $L_{A G}$ on $A G$ which is also a Lie subalgebroid of $\mathbb{T}(A G)$.

It is interesting to observe that since $L_{A G}$ is the Lie algebroid of the $\mathscr{L} \mathscr{A}$ groupoid $L_{G}$, in particular $L_{A G}$ inherits the structure of a double Lie algebroid [Mackenzie 2000]. Double Lie algebroids were introduced by Mackenzie [2011] as a way to understand Drinfeld's doubles of Lie bialgebroids. As a result, multiplicative Dirac structures provide interesting examples of double Lie algebroids.

5D. Dirac groupoids vs. Dirac algebroids. This section is concerned with the statement and proof of the main result of this work. We will prove that, whenever $G$ is a source simply connected Lie groupoid with Lie algebroid $A G$, then the map in Theorem 5.12 is a bijection.

For that, recall that if $M$ is a smooth manifold and $L \subset \mathbb{T} M$ is a Lagrangian subbundle, then there is a well-defined element $\mu_{L} \in \Gamma\left(\bigwedge^{3} L^{*}\right)$ given by

$$
\mu_{L}\left(a_{1}, a_{2}, a_{2}\right):=\left\langle\llbracket a_{1}, a_{2} \rrbracket, a_{3}\right\rangle .
$$

The element $\mu_{L} \in \Gamma\left(\bigwedge^{3} L^{*}\right)$ is referred to as the Courant 3-tensor of $L$. Notice that a Lagrangian subbundle $L \subset \mathbb{T} M$ is a Dirac structure if and only if $\mu_{L}$ vanishes.

Proposition 5.13. Let $G$ be a Lie groupoid over M. Consider a Lagrangian subbundle $L_{G} \subset T G \oplus T^{*} G$, which is also a Lie subgroupoid. Then, the Courant 3-tensor of $L_{G}$ is multiplicative; that is,

$$
\mu_{L_{G}}: \prod_{p_{G} \oplus c_{G}}^{3} L_{G} \rightarrow \mathbb{R}
$$

is a groupoid morphism.

Proof. Let us consider composable pairs $a_{g}^{i}, \bar{a}_{h}^{i}$ in $L_{G}$ with $i=1,2,3$. Set $c_{g h}^{i}=$ $m_{\mathbb{T}}\left(a_{g}^{i}, \bar{a}_{h}^{i}\right) \in\left(L_{G}\right)_{g h}$, for $i=1,2,3$. Choose a section $c^{i} \in \Gamma\left(L_{G}\right)$ such that $c^{i}(g h)=c_{g h}^{i}$. Since $L_{G}$ is a $\mathscr{V} \mathscr{B}$-groupoid, the multiplication on $L_{G}$ is fiberwise surjective. In particular, there exist sections $a^{i}, \bar{a}^{i} \in \Gamma\left(L_{G}\right)$ such that $m_{\mathbb{T}} \circ\left(a^{i}, \bar{a}^{i}\right)=$ $c^{i} \circ m_{G}$, for every $i=1,2,3$. Clearly $a^{i}(g)=a_{g}^{i}$ and $\bar{a}^{i}(h)=\bar{a}_{h}^{i}$, for $i=1,2,3$.

Then,

$$
\begin{aligned}
\mu_{L_{G}}\left(\left(a_{g}^{1}, a_{g}^{2}, a_{g}^{3}\right) *\left(\bar{a}_{h}^{1}, \bar{a}_{h}^{2}, \bar{a}_{h}^{3}\right)\right) & =\mu_{L_{G}}\left(c_{g h}^{1}, c_{g h}^{2}, c_{g h}^{3}\right)=\left\langle\llbracket c^{1}, c^{2} \rrbracket(g h), c^{3}(g h)\right\rangle \\
& =\left\langle m_{\mathbb{}}\left(\llbracket a^{2}, a^{2} \rrbracket, \llbracket \bar{a}^{1}, \bar{a}^{2} \rrbracket\right)(g, h), m_{\mathbb{I}}\left(a^{3}, \bar{a}^{3}\right)(g, h)\right\rangle
\end{aligned}
$$


The last identity follows from the fact that $\left(m_{\mathbb{T}}, m_{G}\right)$ is a Courant morphism (see Proposition 5.2). Now we use the fact that $\langle\cdot, \cdot\rangle_{G}$ is a groupoid morphism to conclude that

$$
\mu_{L_{G}}\left(\left(a_{g}^{1}, a_{g}^{2}, a_{g}^{3}\right) *\left(\bar{a}_{h}^{1}, \bar{a}_{h}^{2}, \bar{a}_{h}^{3}\right)\right)=\mu_{L_{G}}\left(a_{g}^{1}, a_{g}^{2}, a_{g}^{3}\right)+\mu_{L_{G}}\left(\bar{a}_{h}^{1}, \bar{a}_{h}^{2}, \bar{a}_{h}^{3}\right) .
$$

This proves that the function $\mu_{L_{G}}$ is multiplicative.

We would like to describe explicitly the Lie algebroid morphism induced by the multiplicative tensor $\mu_{L_{G}}: \prod_{p_{G} \oplus c_{G}}^{3} L_{G} \rightarrow \mathbb{R}$. For that, we need the next lemma.

Lemma 5.14. Let $M$ be a smooth manifold. Consider a Lagrangian subbundle $L_{M} \subset \mathbb{T} M$. Then, for every $\left(\dot{a}_{1}, \dot{a}_{2}, \dot{a}_{3}\right) \in T L_{M}$ the following identity holds:

$$
T \mu_{L_{M}}\left(\dot{a}_{1}, \dot{a}_{2}, \dot{a}_{3}\right)=\mu_{L_{T M}}\left(\left(J_{M} \oplus \Theta_{M}\right) \dot{a}_{1},\left(J_{M} \oplus \Theta_{M}\right) \dot{a}_{2},\left(J_{M} \oplus \Theta_{M}\right) \dot{a}_{3}\right),
$$

where $L_{T M} \subset \mathbb{T}(T M)$ is the tangent lift of $L_{M}$.

Proof. One has $T \mu_{L_{M}}\left(T a_{1}, T a_{2}, T a_{3}\right)=T\left(\mu_{L_{M}}\left(a_{1}, a_{2}, a_{3}\right)\right)$ for every $a_{1}, a_{2}, a_{3} \in$ $\Gamma_{M}\left(L_{M}\right)$. On the other hand, the canonical map $J_{M} \oplus \Theta_{M}$ applied to each of the sections $T a_{1}, T a_{2}, T a_{3}$ gives $a_{1}^{T}, a_{2}^{T}, a_{3}^{T} \in \Gamma_{T M}\left(L_{T M}\right)$. Thus we conclude that

$$
\mu_{L_{T M}}\left(a_{1}^{T}, a_{2}^{T}, a_{3}^{T}\right)=\left\langle\llbracket a_{1}^{T}, a_{2}^{T} \rrbracket, a_{3}^{T}\right\rangle_{T M}=\left(\left\langle\llbracket a_{1}, a_{2} \rrbracket, a_{3}\right\rangle_{M}\right)^{T},
$$

which is exactly the tangent functor applied to the function $\mu_{L_{M}}\left(a_{1}, a_{2}, a_{3}\right)$. Therefore, for every triple of sections $a_{1}, a_{2}, a_{3}$ of $L_{M}$ we get

$$
T \mu_{L_{M}}\left(T a_{1}, T a_{2}, T a_{3}\right)=\mu_{L_{T M}}\left(a_{1}^{T}, a_{2}^{T}, a_{3}^{T}\right) .
$$

Now we notice, using local coordinates, that for every point $\dot{a} \in T L_{M}$ above $\dot{x} \in T M$ there exists a section $a \in \Gamma_{M}\left(L_{M}\right)$ such that $T a(\dot{x})=\dot{a}$, where $T a \in$ $\Gamma_{T M}\left(T L_{M}\right)$ is the section obtained by applying the tangent functor to the section $a$ of $L_{M}$. This fact together with identity (18) prove the statement.

As a consequence we obtain a direct proof of the Courant integrability of the tangent lift of a Dirac structure $L_{M}$ on $M$.

Corollary 5.15. Let $L_{M}$ be an almost Dirac structure on $M$, and consider the induced almost Dirac structure $L_{T M}$ on TM. Then $L_{T M}$ is Courant integrable if $L_{M}$ is Courant integrable.

Now consider a multiplicative Dirac structure $L_{G}$ on $G$. The application of the Lie functor to the groupoid morphism $\mu_{L_{G}}$ of Proposition 5.13 yields a Lie algebroid morphism

$$
A\left(\mu_{L_{G}}\right): \prod_{A\left(p_{G} \oplus c_{G}\right)}^{3} A\left(L_{G}\right) \rightarrow \mathbb{R} .
$$

Since $A\left(\mu_{L_{G}}\right)=\left.T \mu_{L_{G}}\right|_{A\left(L_{G}\right)}$, we conclude: 
Proposition 5.16. For the Lagrangian subbundle $L_{A G}=\left(j_{G}^{-1} \oplus j_{G}^{\prime}\right) A\left(L_{G}\right) \subseteq$ $\mathbb{T}(A G)$, we have

$$
A\left(\mu_{L_{G}}\right)=\mu_{L_{A G}} \circ\left(j_{G}^{-1} \oplus j_{G}^{\prime}\right)^{(3)},
$$

where $\left(j_{G}^{-1} \oplus j_{G}^{\prime}\right)^{(3)}: \prod_{A\left(p_{G} \oplus c_{G}\right)}^{3} A\left(L_{G}\right) \rightarrow \prod_{p_{A G} \oplus c_{A G}}^{3} L_{A G}$ denotes the natural extension of $\left(j_{G}^{-1} \oplus j_{G}^{\prime}\right)$.

Proof. This follows directly from Lemma 5.14 and the fact that $j_{G}$ and $j_{G}^{\prime}$ are suitable restrictions of $J_{G}$ and $\Theta_{G}$, respectively.

Now we are ready to state the main theorem of this work.

Theorem 5.17. Let $G$ be a source simply connected Lie groupoid with Lie algebroid AG. There is a one-to-one correspondence between multiplicative Dirac structures on $G$ and morphic Dirac structures on AG. The correspondence is given by the map in Theorem 5.12.

Proof. Let $L_{G}$ be a multiplicative Dirac structure on $G$. Consider the Lagrangian subbundle $L_{A G}:=\left(j_{G}^{-1} \oplus j_{G}^{\prime}\right)\left(A\left(L_{G}\right)\right) \subset \mathbb{T} A G$. Since $\mu_{L_{G}} \equiv 0$, then Proposition 5.16 implies that $\mu_{L_{A G}} \equiv 0$. Thus, $L_{A G}$ is a Dirac structure on $A G$ which is clearly morphic. Notice that the integrability of $L_{A G}$ is also a consequence of Theorem 5.12. Conversely, consider an element $L_{A} \in \operatorname{Dir}_{\text {morph }}(A G)$; thus $L_{A}$ is a linear Dirac structure on $A G$ such that $L_{A} \subseteq \mathbb{T} A G$ is a $\mathscr{V} \mathscr{P}$-subalgebroid. Since $G$ is source simply connected, $\mathbb{T} G$ is the source simply connected Lie groupoid which integrates the Lie algebroid $\mathbb{T} A G$. As explained in [Bursztyn et al. $\geq 2013$ ], the $\mathscr{V} \mathscr{B}$-subalgebroid $L_{A} \subseteq \mathbb{T} A$ integrates to a source simply connected $\mathscr{V} \mathscr{B}$-subgroupoid $L_{G} \subseteq \mathbb{T} G$. We will prove that $L_{G}$ is a multiplicative Dirac structure on $G$. Since $L_{A G} \subseteq \mathbb{T} A G$ is Lagrangian with respect to the canonical symmetric pairing $\langle\cdot, \cdot\rangle_{A G}$ on $\mathbb{T} A G$, we conclude from Proposition 5.11 that $L_{G}$ is Lagrangian with respect to the canonical symmetric pairing $\langle\cdot, \cdot\rangle_{G}$ on $\mathbb{T} G$. It remains to show that $L_{G} \subseteq \mathbb{T} G$ is integrable with respect to the Courant bracket. Equivalently, we have to prove that the Courant 3-tensor $\mu_{L_{G}} \in \Gamma\left(\bigwedge^{3} L_{G}^{*}\right)$ is zero. Since $L_{A} \subseteq \mathbb{\mathbb { N }} A G$ is a Dirac structure, the induced Courant 3-tensor $\mu_{L_{A}} \in \Gamma\left(\bigwedge^{3} L_{A}^{*}\right)$ vanishes. Therefore, combining Proposition 5.16 (applied to the zero Lie algebroid morphism) with Lie's second theorem we conclude that $\mu_{L_{G}} \equiv 0$, as desired. This shows that $L_{G}$ is a Dirac structure on $G$, which by definition is multiplicative.

Remark 5.18. Theorem 5.17 provides a direct proof of the Notice that integrability of the Lagrangian subbundle $L_{A G} \subset \mathbb{T}(A G)$ associated to a multiplicative Dirac structure $L_{G} \subset \mathbb{T} G$, without using the theory of $\mathscr{L} \mathscr{A}$-groupoids. In spite of this, we believe that the fact that $L_{A G}$ inherits the structure of a double Lie algebroid is interesting in itself. This relies on the observation that $L_{G}$ is an $\mathscr{L} \mathscr{A}$-groupoid. 
5E. Main examples revisited. We have shown several examples of Dirac groupoids and Dirac algebroids. See Sections 3 and 4, respectively. Here we will see that both classes of examples are related by the construction explained in Section 5C. Throughout this subsection $G$ denotes a Lie groupoid over $M$ with Lie algebroid $A G$.

5E1. Poisson groupoids and Lie bialgebroids. Consider a multiplicative Poisson bivector $\pi_{G}$ on $G$. It is well known that in this case $M \subseteq G$ is a coisotropic submanifold and, in particular, the conormal bundle $N^{*} M \cong A^{*} G$ inherits a Lie algebroid structure. The Dirac structure $L_{G}$ on $G$ defined by the graph of $\pi_{G}$ is a multiplicative Dirac structure. The multiplicativity of this Dirac structure is equivalent to $\pi_{G}^{\sharp}: T^{*} G \rightarrow T G$ being a morphism of Lie groupoids, and the associated Lie algebroid morphism coincides, up to identifications, with $\pi_{A G}^{\sharp}$ : $T^{*}(A G) \rightarrow T(A G)$, where $\pi_{A G}$ denotes the linear Poisson bivector on $A G$ dual to the Lie algebroid $A^{*} G$. One concludes that the corresponding Dirac structure $L_{A G}$ on $A G$ is exactly the graph of $\pi_{A G}$. Since $L_{A G}$ is a Lie subalgebroid of $\mathbb{T} A G$, the bundle map $\pi_{A G}^{\sharp}: T^{*}(A G) \rightarrow T(A G)$ is a Lie algebroid morphism. This is equivalent to saying that $\left(A G, A^{*} G\right)$ is a Lie bialgebroid. As a corollary of Theorem 5.17 we obtain:

Corollary 5.19 [Mackenzie and Xu 2000]. Let G be a source simply connected Lie groupoid with Lie algebroid AG. There is a one-to-one correspondence between multiplicative Poisson bivectors on $G$ and Lie bialgebroid structures on $\left(A G, A^{*} G\right)$.

5E2. Multiplicative 2-forms and IM-2-forms. Assume that $\omega_{G} \in \Omega^{2}(G)$ is a multiplicative closed 2-form on $G$. The Dirac structure $L_{G}$ given by the graph of $\omega_{G}^{\sharp}: T G \rightarrow T^{*} G$ is multiplicative. In this case, the corresponding Dirac structure $L_{A G}$ on $A G$ is given by the graph of the closed 2-form $\omega_{A G}:=-\sigma^{*} \omega_{c a n}$, where $\sigma: A G \rightarrow T^{*} M$ is defined by $\sigma(u)=\left.i_{u} \omega_{G}\right|_{T M}$. Since the Dirac structure $L_{A G}$ is a Lie subalgebroid of $\mathbb{T}(A G)$, we conclude that the bundle map $\omega_{A G}^{\sharp}: T(A G) \rightarrow T^{*}(A G)$ is a Lie algebroid morphism. As shown in [Bursztyn et al. 2009a], this is equivalent to the bundle map $\sigma: A G \rightarrow T^{*} M$ being an IM-2-form on $A G$; that is, for every $u, v \in \Gamma(A G)$, the following conditions hold:

- $\left\langle\sigma(u), \rho_{A G}(v)\right\rangle=-\left\langle\sigma(v), \rho_{A G}(u)\right\rangle$;

- $\sigma[u, v]=\mathscr{L}_{\rho_{A G}(u)} \sigma(v)-\mathscr{L}_{\rho_{A G}(v)} \sigma(u)+d\left\langle\sigma(u), \rho_{A G}(v)\right\rangle$.

As a corollary of Theorem 5.17, we get:

Corollary 5.20 [Bursztyn et al. 2004]. Let $G$ be a source simply connected Lie groupoid with Lie algebroid AG. There is a one-to-one correspondence between multiplicative closed 2-forms on $G$ and IM-2-forms on AG.

5E3. Foliated groupoids and foliated algebroids. Let $F_{G} \subseteq T G$ be a multiplicative involutive subbundle. Then, the Dirac structure $L_{G}=F_{G} \oplus F_{G}^{\circ}$ is multiplicative. 
The corresponding Dirac structure $L_{A G}$ on $A G$ associated to $L_{G}$ is given by $L_{A G}=$ $F_{A G} \oplus F_{A G}^{\circ} \subset \mathbb{T}(A G)$, where $F_{A G}:=j_{G}^{-1}\left(A\left(F_{G}\right)\right) \subseteq T(A G)$. Since $L_{A G}$ is a Dirac structure which is also a Lie subalgebroid of $\mathbb{T}(A G)$, we conclude that $F_{A G} \subseteq T(A G)$ is an involutive subbundle which is also a Lie subalgebroid of $T(A G) \rightarrow T M$. We refer to such a subbundle as a morphic foliation on $A G$. As a corollary of Theorem 5.17, we obtain the next result.

Corollary 5.21 [Hawkins 2008]. Let $G$ be a source simply connected Lie groupoid with Lie algebroid AG. There exists a one-to-one correspondence between multiplicative foliations on $G$ and morphic foliations on $A G$.

As shown in [Hawkins 2008; Jotz and Ortiz 2012], having a morphic foliation on $A G$ is equivalent to $A G$ be equipped with an IM-foliation, that is, a triple $\left(F_{M}, K, \nabla\right)$ where $F_{M} \subseteq T M$ is an involutive subbundle, $K \subseteq A G$ is a Lie subalgebroid with $\rho_{A G}(K) \subseteq F_{M}$, and $\nabla$ is an $F_{M}$-connection on $A G / K$ satisfying the following conditions:

- $\nabla$ is flat.

- If $u \in \Gamma(A G)$ satisfies $\nabla_{\Gamma\left(F_{M}\right)}(u+K) \in \Gamma(K)$, then $[u, \Gamma(K)] \subseteq \Gamma(K)$.

- If $u, v \in \Gamma(A G)$ are such that $\nabla_{\Gamma\left(F_{M}\right)}(u+K), \nabla_{\Gamma\left(F_{M}\right)}(v+K) \in \Gamma(K)$, it follows that $\nabla_{\Gamma\left(F_{M}\right)}([u, v]+K) \in \Gamma(K)$.

- If $u \in \Gamma(A G)$ satisfies $\nabla_{\Gamma\left(F_{M}\right)}(u+K) \in \Gamma(K)$, then $\left[\rho_{A G}(u), \Gamma\left(F_{M}\right)\right] \subseteq$ $\Gamma\left(F_{M}\right)$.

The properties as above determine completely the morphic foliation $F_{A G}$ on $A G$. In particular, Dirac structures of the form $L_{A G}=F_{A G} \oplus F_{A G}^{\circ}$ are in one-to-one correspondence with IM-foliations. Additionally, there exists a conceptually clear interpretation of IM-foliations in terms of representations up to homotopy. See [Drummond et al. 2013] for more details.

5E4. Dirac Lie groups and Dirac Lie algebras. Let $G$ be a Lie group with Lie algebra $\mathfrak{g}$ and let $L_{G} \in \operatorname{Dir}_{\text {mult }}(G)$ be a multiplicative Dirac structure. Consider the Dirac structure $L_{\mathfrak{g}}$ on $\mathfrak{g}$ associated to $L_{G}$. It was shown in [Ortiz 2008] that $\operatorname{ker}\left(L_{G}\right):=L_{G} \cap T G$ is a regular involutive subbundle of $T G$, in particular $\operatorname{ker}\left(L_{\mathfrak{g}}\right)=$ $j_{G}^{-1}\left(A\left(\operatorname{ker}\left(L_{G}\right)\right)\right)$ is an involutive subbundle of $T \mathfrak{g}$. Since $\operatorname{ker}\left(L_{\mathfrak{g}}\right)$ is a linear foliation on $\mathfrak{g}$, that is, multiplicative with respect to the abelian group structure on $\mathfrak{g}$, then the leaf through $0 \in \mathfrak{g}$ is a vector subspace $\mathfrak{h} \subseteq \mathfrak{g}$. The other leaves are affine subspaces of $\mathfrak{g}$ modeled on $\mathfrak{h}$. In particular, the space of characteristic leaves of $L_{\mathfrak{g}}$ coincides with the quotient space $\mathfrak{g} / \mathfrak{h}$. The fact that $L_{g} \subseteq \mathbb{T} \mathfrak{g}$ is a Lie subalgebroid implies that $\mathfrak{h} \subseteq \mathfrak{g}$ is an ideal. Therefore, the space of characteristic leaves $\mathfrak{g} / \mathfrak{h}$ of $L_{\mathfrak{g}}$ inherits a unique Lie algebra structure making the quotient map $\phi: \mathfrak{g} \rightarrow \mathfrak{g} / \mathfrak{h}$ into a surjective Lie algebra morphism. Since $\mathfrak{g} / \mathfrak{h}$ is the space of characteristic 
leaves of $L_{\mathfrak{g}}$, there is a unique Poisson structure $\pi$ on $\mathfrak{g} / \mathfrak{h}$ making the quotient map $\phi: \mathfrak{g} \rightarrow \mathfrak{g} / \mathfrak{h}$ into a forward and backward Dirac map. Since $L_{\mathfrak{g}}$ is a morphic Dirac structure, we conclude that $\pi$ is a morphic bivector on $\mathfrak{g} / \mathfrak{h}$. In particular, the pair $\left(\mathfrak{g} / \mathfrak{h},(\mathfrak{g} / \mathfrak{h})^{*}\right)$ is a Lie bialgebra. Conversely, given a Lie algebra $\mathfrak{g}$ and an ideal $\mathfrak{h} \subseteq \mathfrak{g}$ such that $\left(\mathfrak{g} / \mathfrak{h},(\mathfrak{g} / \mathfrak{h})^{*}\right)$ is a Lie bialgebra, then the linear Poisson bivector $\pi$ on $\mathfrak{g} / \mathfrak{h}$ is morphic. The surjective Lie algebra morphism $\mathfrak{g} \rightarrow \mathfrak{g} / \mathfrak{h}$ induces a Dirac structure $L_{\mathfrak{g}}$ on $\mathfrak{g}$ (the pull-back of $\pi$ ) which is morphic as well. We have proved the following result.

Proposition 5.22. Let $\mathfrak{g}$ be a finite-dimensional Lie algebra. There is a one-to-one correspondence between

(1) morphic Dirac structures on $\mathfrak{g}$, and

(2) ideals $\mathfrak{h} \subseteq \mathfrak{g}$ such that $\left(\mathfrak{g} / \mathfrak{h},(\mathfrak{g} / \mathfrak{h})^{*}\right)$ is a Lie bialgebra.

The proposition above recovers the results of [Ortiz 2008].

5E5. Tangent lifts of Dirac structures. Let $L_{G}$ be a multiplicative Dirac structure on $G$. Consider the associated morphic Dirac structure $L_{A G}$ on the Lie algebroid of $G$. We can lift $L_{G}$ to a multiplicative Dirac structure on the tangent groupoid $T G$. Similarly, as explained in Section 4B3, the morphic Dirac structure $L_{A G}$ can be lifted to a morphic Dirac structure $L_{T(A G)}$ on the tangent Lie algebroid $T(A G) \rightarrow T M$. It is straightforward to check that the morphic Dirac structure on $T(A G)$ associated to $L_{T G}$ as in Theorem 5.17 coincides with the tangent lift $L_{T(A G)}$ of $L_{A G}$. That is, the tangent functor commutes with the Lie functor.

5E6. Symmetries of Dirac groupoids. Let $L_{G}$ be a multiplicative Dirac structure on $G$. Consider the associated morphic Dirac structure $L_{A G}$ on $A G$ as in Theorem 5.17. Let $H$ be a Lie group acting freely and properly on $G$ by groupoid automorphisms $\Phi_{h}: G \rightarrow G, h \in H$. Applying the Lie functor to each $\Phi_{h}: G \rightarrow G$ yields a free and proper $H$-action on $A G$ by Lie algebroid automorphisms $A\left(\Phi_{h}\right): A G \rightarrow A H, h \in H$. Assume that the $H$-orbits of $G$ coincide with the characteristic leaves of $L_{G}$. Then, the $H$-orbits of $A G$ coincide with the characteristic leaves of $L_{A G}$. We have shown that in this situation we can endow the space of characteristic leaves $G / H$ of $L_{G}$ with a unique multiplicative Poisson bivector $\pi_{G / H}$ making the quotient map $G \rightarrow G / H$ into a forward and backward Dirac map. Similarly, the space of characteristic leaves $A G / H$ of $L_{A G}$ inherits a unique morphic Poisson structure $\pi_{A G / H}$ making the quotient map $A G \rightarrow A G / H$ into a forward and backward Dirac map. One can easily see that the morphic Dirac structure $L_{A G / H}$ associated to $\pi_{G / H}$ as in Section 5E1 coincides with the morphic Dirac structure on $A G / H$ given by the graph of $\pi_{A G / H}$. As a consequence, the Lie bialgebroid of $\left(G / H, \pi_{G / H}\right)$ is exactly $\left(A G / H,(A G / H)^{*}\right)$. 
5E7. B-field transformations. Let $L_{G}$ be a multiplicative Dirac structure on $G$. Assume that $B_{G}$ is a multiplicative closed 2-form on $G$. Consider the Dirac structure $L_{G}^{B}$ on $G$, obtained out of $L_{G}$ by applying the $B$-field transformation with respect to $B_{G}$. As observed in [Bursztyn et al. 2009a], every multiplicative closed 2-form on $G$ induces a morphic closed 2-form $B_{A G}$ on $A G$. A direct computation shows that the morphic Dirac structure $L_{A G}^{B}$ corresponding to $L_{G}^{B}$ (as in Theorem 5.17) is given by the $B$-field transformation of $L_{A G}$ with respect to $B_{A G}$, in agreement with [Ortiz 2012].

5E8. Generalized complex groupoids. Let $L_{G} \subseteq \mathbb{T}_{\mathbb{C}} G$ be a multiplicative generalized complex structure on $G$. The construction explained in Theorem 5.12 applies also to the case of multiplicative generalized complex structures. As a result, there is a morphic Dirac structure $L_{A G} \subseteq \mathbb{T}_{\mathbb{C}} A G$ given by $L_{A G}:=\left(j_{G}^{-1} \oplus j_{G}^{\prime}\right){ }_{\mathbb{C}}\left(A\left(L_{G}\right)\right)$, where $\left(j_{G}^{-1} \oplus j_{G}^{\prime}\right)_{\mathbb{C}}: A\left(\mathbb{T}_{\mathbb{C}} G\right) \rightarrow \mathbb{T}_{\mathbb{C}}(A G)$ denotes the complexification of the canonical isomorphism $\left(j_{G}^{-1} \oplus j_{G}^{\prime}\right): A(\mathbb{T} G) \rightarrow \mathbb{T}(A G)$. Observe that $L_{A G} \subseteq \mathbb{T}_{\mathbb{C}} A G$ is in fact a generalized complex structure making the pair $\left(A G, L_{A G}\right)$ into a generalized Lie algebroid. For that, we only need to check that $L_{A G} \cap \overline{L_{A G}}=\{0\}$. Indeed, one easily checks that the conjugation map $\overline{(\cdot)}_{G}: \mathbb{T}_{\mathbb{C}} G \rightarrow \mathbb{T}_{\mathbb{C}} G$ is a Lie groupoid isomorphism. Therefore, the generalized complex structure $\bar{L}_{G}$ on $G$ is also multiplicative. Since $\mathbb{T}_{\mathbb{C}} G=L_{G} \oplus \bar{L}_{G}$, the application of the Lie functor yields a decomposition

$$
A\left(\mathbb{T}_{\mathbb{C}} G\right)=A\left(L_{G}\right) \oplus A\left(\bar{L}_{G}\right) .
$$

Straightforward computation shows that the Lie algebroid isomorphism $A\left(\overline{(\cdot)}_{G}\right)$ : $A\left(\mathbb{T}_{\mathbb{C}} G\right) \rightarrow A\left(\mathbb{T}_{\mathbb{C}} G\right)$ satisfies

$$
\left(j_{G}^{-1} \oplus j_{G}^{\prime}\right)_{\mathbb{C}} \circ A\left({\overline{(\cdot)_{G}}}_{G}\right)=\overline{(\cdot)}_{A G},
$$

where the map of the right hand side of the identity above is the conjugation map $\mathbb{T}_{\mathbb{C}}(A G) \rightarrow \mathbb{T}_{\mathbb{C}}(A G)$. Hence, applying the canonical isomorphism $\left(j_{G}^{-1} \oplus j_{G}^{\prime}\right)_{\mathbb{C}}:$ $A\left(\mathbb{T}_{\mathbb{C}} G\right) \rightarrow \mathbb{T}_{\mathbb{C}}(A G)$ on both sides of (19), gives rise to

$$
\mathbb{T}_{\mathbb{C}} A G=L_{A G} \oplus \bar{L}_{A G} .
$$

Therefore, $L_{A G}$ is transversal to $\bar{L}_{A G}$ and we conclude that $L_{A G}$ is a morphic generalized complex structure. In this situation, Theorem 5.17 gives rise to the following result.

Proposition 5.23 [Jotz et al. 2012]. Let $G$ be a source simply connected Lie groupoid with Lie algebroid AG. There is a one-to-one correspondence between multiplicative generalized complex structures on $G$ and morphic generalized complex structures on $A G$. 


\section{Conclusions and final remarks}

This work can be considered as the first step toward describing multiplicative Dirac structures infinitesimally. We have seen that every multiplicative Dirac structure $L_{G}$ on a Lie groupoid $G$ induces a Dirac structure $L_{A G}$ on its Lie algebroid $A G$ which is compatible with the algebroid structure in the sense that $L_{A G} \subseteq \mathbb{T}(A G)$ is a Lie subalgebroid. Notice that in the special situation of Poisson groupoids (resp. multiplicative closed 2-forms, multiplicative foliations) the induced Dirac structure on $A G$ is equivalent to endowing $\left(A G, A^{*} G\right)$ with a Lie bialgebroid structure (resp. IM-2-form, IM-foliation). Therefore, it would be interesting to introduce a suitable notion of IM-Dirac structure, providing a more explicit description of Dirac structures compatible with a Lie algebroid, unifying different infinitesimal structures such as Lie bialgebroids, IM-2-forms and IM-foliations. This study will be part of a future work.

\section{Acknowledgements}

The content of this work is based on the author's doctoral thesis [Ortiz 2009] carried out at IMPA under the supervision of Henrique Bursztyn. The author would like to thank Henrique Bursztyn for suggesting the problem of studying multiplicative Dirac structures as well as for several conversations and suggestions that have improved this work. While the author was at IMPA, he was supported by a PEC-PG doctoral scholarship from CAPES (Brazil). Finally, the author would like to thank the referee for all comments and suggestions that have improved the presentation of this paper.

\section{References}

[Alekseev and Xu 2011] A. Alekseev and P. Xu, "Derived brackets and Courant algebroids", preprint, 2011, Available at http://www.math.psu.edu/ping/anton-final.pdf.

[Arias Abad and Crainic 2011] C. Arias Abad and M. Crainic, "The Weil algebra and the Van Est isomorphism", Ann. Inst. Fourier (Grenoble) 61:3 (2011), 927-970. MR 2918722 Zbl 1237.58021

[Boumaiza and Zaalani 2009] M. Boumaiza and N. Zaalani, "Relèvement d'une algébroïde de Courant”, C. R. Math. Acad. Sci. Paris 347:3-4 (2009), 177-182. MR 2010m:53123 Zbl 1161.53073

[Bursztyn 2005] H. Bursztyn, "On gauge transformations of Poisson structures", pp. 89-112 in Quantum field theory and noncommutative geometry, edited by U. Carow-Watamura et al., Lecture Notes in Phys. 662, Springer, Berlin, 2005. MR 2006g:53127 Zbl 1076.53104

[Bursztyn and Crainic 2009] H. Bursztyn and M. Crainic, "Dirac geometry, quasi-Poisson actions and $D / G$-valued moment maps", J. Differential Geom. 82:3 (2009), 501-566. MR 2010h:53131 Zbl 1177.53072

[Bursztyn and Radko 2003] H. Bursztyn and O. Radko, "Gauge equivalence of Dirac structures and symplectic groupoids", Ann. Inst. Fourier (Grenoble) 53:1 (2003), 309-337. MR 2004d:53101 Zbl 1026.58019 
[Bursztyn et al. 2004] H. Bursztyn, M. Crainic, A. Weinstein, and C. Zhu, "Integration of twisted Dirac brackets”, Duke Math. J. 123:3 (2004), 549-607. MR 2005e:53128 Zbl 1067.58016

[Bursztyn et al. 2009a] H. Bursztyn, A. Cabrera, and C. Ortiz, "Linear and multiplicative 2-forms", Lett. Math. Phys. 90:1-3 (2009), 59-83. MR 2011a:58038 Zbl 1206.58005

[Bursztyn et al. 2009b] H. Bursztyn, D. Iglesias Ponte, and P. Ševera, "Courant morphisms and moment maps”, Math. Res. Lett. 16:2 (2009), 215-232. MR 2010f:53152 Zbl 1183.53076

[Bursztyn et al. $\geq 2013$ ] H. Bursztyn, A. Cabrera, and M. del Hoyo, "On the integration of $\mathscr{V} \mathscr{B}$ algebroids". In preparation.

[Cattaneo and Felder 2001] A. S. Cattaneo and G. Felder, "Poisson sigma models and symplectic groupoids", pp. 61-93 in Quantization of singular symplectic quotients, edited by N. P. Landsman et al., Progr. Math. 198, Birkhäuser, Basel, 2001. MR 2003h:53116 Zbl 1038.53074

[Coste et al. 1987] A. Coste, P. Dazord, and A. Weinstein, "Groupoïdes symplectiques", Publ. Dép. Math. Nouvelle Sér. 87-2, Univ. Claude-Bernard, Lyon, 1987. MR 90g:58033 Zbl 0668.58017

[Courant 1990a] T. Courant, “Tangent Dirac structures", J. Phys. A 23:22 (1990), 5153-5168. MR 92d:58064 Zbl 0715.58013

[Courant 1990b] T. J. Courant, "Dirac manifolds", Trans. Amer. Math. Soc. 319:2 (1990), 631-661. MR 90m:58065 Zbl 0850.70212

[Courant and Weinstein 1988] T. Courant and A. Weinstein, "Beyond Poisson structures", pp. 39-49 in Action hamiltoniennes de groupes: Troisième théorème de Lie (Lyon, 1986), edited by P. Dazord et al., Travaux en Cours 27, Hermann, Paris, 1988. MR 89h:58054 Zbl 0698.58020

[Crainic et al. 2012] M. Crainic, M. A. Salazar, and I. Struchiner, "Multiplicative forms and Spencer operators", preprint, 2012. arXiv 1210.2277

[Drinfel'd 1983] V. G. Drinfel'd, "Hamiltonian structures on Lie groups, Lie bialgebras and the geometric meaning of classical Yang-Baxter equations", Dokl. Akad. Nauk SSSR 268:2 (1983), 285287. In Russian; translated in Soviet Math. Dokl. 27:1 (1983), 68-71. MR 84i:58044 Zbl 0526.58017

[Drummond et al. 2013] T. Drummond, M. Jotz, and C. Ortiz, “ $\mathscr{Q} \mathscr{B}$-algebroid morphisms and representations up to homotopy", preprint, 2013. arXiv 1302.3987

[Etingof and Varchenko 1998] P. Etingof and A. Varchenko, "Geometry and classification of solutions of the classical dynamical Yang-Baxter equation", Comm. Math. Phys. 192:1 (1998), 77-120. MR 99e:32032 Zbl 0915.17018

[Fernandes and Iglesias $\geq 2013$ ] R. L. Fernandes and D. Iglesias, "Symmetries and reduction of Poisson Lie groupoids". In preparation.

[Grabowska and Grabowski 2011] K. Grabowska and J. Grabowski, "Dirac algebroids in Lagrangian and Hamiltonian mechanics”, J. Geom. Phys. 61:11 (2011), 2233-2253. MR 2012h:53183 Zbl 1223.37064

[Grabowski and Urbański 1995] J. Grabowski and P. Urbański, “Tangent lifts of Poisson and related structures”, J. Phys. A 28:23 (1995), 6743-6777. MR 96m:58080 Zbl 0872.58028

[Grabowski and Urbański 1997] J. Grabowski and P. Urbański, “Tangent and cotangent lifts and graded Lie algebras associated with Lie algebroids", Ann. Global Anal. Geom. 15:5 (1997), 447-486. MR 99k:58199 Zbl 0973.58006

[Gualtieri 2003] M. Gualtieri, Generalized complex geometry, Ph.D. thesis, Oxford University, 2003. arXiv math/0401221

[Hawkins 2008] E. Hawkins, “A groupoid approach to quantization”, J. Symplectic Geom. 6:1 (2008), 61-125. MR 2009j:46167 Zbl 1154.46041 
[Higgins and Mackenzie 1990] P. J. Higgins and K. Mackenzie, "Algebraic constructions in the category of Lie algebroids", J. Algebra 129:1 (1990), 194-230. MR 92e:58241 Zbl 0696.22007

[Hitchin 2003] N. Hitchin, "Generalized Calabi-Yau manifolds", Q. J. Math. 54:3 (2003), 281-308. MR 2004h:32024 Zbl 1076.32019

[Jotz and Ortiz 2012] M. Jotz and C. Ortiz, "Foliated groupoids and their infinitesimal data", preprint, 2012. arXiv 1109.4515

[Jotz et al. 2012] M. Jotz, M. Stiénon, and P. Xu, “Glanon groupoids”, preprint, 2012. arXiv 1109.5011 [Konieczna and Urbański 1999] K. Konieczna and P. Urbański, "Double vector bundles and duality", Arch. Math. (Brno) 35:1 (1999), 59-95. MR 2000b:53110 Zbl 1054.53055

[Li-bland 2012] D. Li-bland, LAA-Courant algebroids and their applications, Ph.D. thesis, University of Toronto, 2012. arXiv 1204.2796

[Li-Bland and Meinrenken 2011] D. Li-Bland and E. Meinrenken, "Dirac Lie groups", preprint, 2011. arXiv $1110.1525 \mathrm{v} 1$

[Li-Bland and Ševera 2011] D. Li-Bland and P. Ševera, "Quasi-Hamiltonian groupoids and multiplicative Manin pairs”, Int. Math. Res. Not. 2011:10 (2011), 2295-2350. MR 2012h:53184 Zbl 1218.53083

[Mackenzie 1992] K. C. H. Mackenzie, "Double Lie algebroids and second-order geometry, I", Adv. Math. 94:2 (1992), 180-239. MR 93f:58255 Zbl 0765.57025

[Mackenzie 2000] K. C. H. Mackenzie, "Double Lie algebroids and second-order geometry, II", Adv. Math. 154:1 (2000), 46-75. MR 2002f:58025 Zbl 0971.58015

[Mackenzie 2005] K. C. H. Mackenzie, General theory of Lie groupoids and Lie algebroids, London Mathematical Society Lecture Note Series 213, Cambridge University Press, 2005. MR 2006k:58035 Zbl 1078.58011

[Mackenzie 2011] K. C. H. Mackenzie, "Ehresmann doubles and Drinfel'd doubles for Lie algebroids and Lie bialgebroids", J. Reine Angew. Math. 658 (2011), 193-245. MR 2012g:53169 Zbl 1246.53112

[Mackenzie and Xu 1994] K. C. H. Mackenzie and P. Xu, "Lie bialgebroids and Poisson groupoids", Duke Math. J. 73:2 (1994), 415-452. MR 95b:58171 Zbl 0844.22005

[Mackenzie and Xu 2000] K. C. H. Mackenzie and P. Xu, "Integration of Lie bialgebroids", Topology 39:3 (2000), 445-467. MR 2001b:53104 Zbl 0961.58009

[Mehta 2009] R. A. Mehta, " $Q$-groupoids and their cohomology", Pacific J. Math. 242:2 (2009), 311-332. MR 2010j:22005 Zbl 1185.22002

[Ortiz 2008] C. Ortiz, "Multiplicative Dirac structures on Lie groups", C. R. Math. Acad. Sci. Paris 346:23-24 (2008), 1279-1282. MR 2010a:53177 Zbl 1163.53052

[Ortiz 2009] C. Ortiz, Multiplicative Dirac structures, Ph.D. thesis, IMPA, 2009, Available at https:// dl.dropboxusercontent.com/u/56977129/IMPAthesis.pdf.

[Ortiz 2012] C. Ortiz, “ $B$-field transformations of Poisson groupoids”, preprint, 2012. To appear in Matemática Contemporânea, Proceedings of the Second Latin Congress on Symmetries in Geometry and Physics. arXiv 1107.3343

[Pradines 1974] J. Pradines, "Représentation des jets non holonomes par des morphismes vectoriels doubles soudés", C. R. Acad. Sci. Paris Sér. A 278 (1974), 1523-1526. MR 52 \#9268 Zbl 0285.58002

[Tang 2006] X. Tang, "Deformation quantization of pseudo-symplectic (Poisson) groupoids", Geom. Funct. Anal. 16:3 (2006), 731-766. MR 2007b:53187 Zbl 1119.53061

[Tulczyjew 1977] W. M. Tulczyjew, “The Legendre transformation”, Ann. Inst. H. Poincaré Sect. A (N.S.) 27:1 (1977), 101-114. MR 58 \#13177 Zbl 0365.58011 
[Vaisman 2005] I. Vaisman, "Tangent Dirac structures and submanifolds”, Int. J. Geom. Methods Mod. Phys. 2:5 (2005), 759-775. MR 2006j:53123 Zbl 1081.53071

[Weinstein 1987] A. Weinstein, "Symplectic groupoids and Poisson manifolds", Bull. Amer. Math. Soc. (N.S.) 16:1 (1987), 101-104. MR 88c:58019 Zbl 0618.58020

[Weinstein 1988] A. Weinstein, "Coisotropic calculus and Poisson groupoids", J. Math. Soc. Japan 40:4 (1988), 705-727. MR 90b:58091 Zbl 0642.58025

[Weinstein and Xu 1991] A. Weinstein and P. Xu, "Extensions of symplectic groupoids and quantization”, J. Reine Angew. Math. 417 (1991), 159-189. MR 92k:58094 Zbl 0722.58021

[Yano and Ishihara 1973] K. Yano and S. Ishihara, Tangent and cotangent bundles: Differential geometry, Pure and Applied Mathematics 16, Marcel Dekker, New York, 1973. MR 50 \#3142 Zbl 0262.53024

Received December 12, 2012. Revised May 14, 2013.

CRISTIÁN ORTIZ

DePartamento de MATEMÁtica

UNIVERSIDADE FEDERAL DO PARANÁ

Setor de CiênCias Exatas - Centro Politécnico

81531-990 CURITIBA

BRAZIL

cristian.ortiz@ufpr.br 


\title{
PACIFIC JOURNAL OF MATHEMATICS
}

\author{
msp.org/pjm
}

Founded in 1951 by E. F. Beckenbach (1906-1982) and F. Wolf (1904-1989)

\section{EDITORS}

V. S. Varadarajan (Managing Editor)

Department of Mathematics

University of California

Los Angeles, CA 90095-1555

pacific@math.ucla.edu

Paul Balmer

Department of Mathematics

University of California

Los Angeles, CA 90095-1555

balmer@math.ucla.edu

Daryl Cooper

Department of Mathematics

University of California

Santa Barbara, CA 93106-3080 cooper@math.ucsb.edu

Jiang-Hua Lu

Department of Mathematics

The University of Hong Kong

Pokfulam Rd., Hong Kong jhlu@maths.hku.hk
Don Blasius

Department of Mathematics University of California

Los Angeles, CA 90095-1555

blasius@math.ucla.edu

Robert Finn

Department of Mathematics Stanford University

Stanford, CA 94305-2125

finn@math.stanford.edu

Sorin Popa

Department of Mathematics

University of California

Los Angeles, CA 90095-1555 popa@math.ucla.edu

Paul Yang

Department of Mathematics Princeton University

Princeton NJ 08544-1000

yang@math.princeton.edu

\section{PRODUCTION}

Silvio Levy, Scientific Editor, production@msp.org

\section{SUPPORTING INSTITUTIONS}

ACADEMIA SINICA, TAIPEI

CALIFORNIA INST. OF TECHNOLOGY

INST. DE MATEMÁTICA PURA E APLICADA

KEIO UNIVERSITY

MATH. SCIENCES RESEARCH INSTITUTE

NEW MEXICO STATE UNIV.

OREGON STATE UNIV.

\author{
STANFORD UNIVERSITY \\ UNIV. OF BRITISH COLUMBIA \\ UNIV. OF CALIFORNIA, BERKELEY \\ UNIV. OF CALIFORNIA, DAVIS \\ UNIV. OF CALIFORNIA, LOS ANGELES \\ UNIV. OF CALIFORNIA, RIVERSIDE \\ UNIV. OF CALIFORNIA, SAN DIEGO \\ UNIV. OF CALIF., SANTA BARBARA
}

\author{
Vyjayanthi Chari \\ Department of Mathematics \\ University of California \\ Riverside, CA 92521-0135 \\ chari@math.ucr.edu \\ Kefeng Liu \\ Department of Mathematics \\ University of California \\ Los Angeles, CA 90095-1555 \\ liu@math.ucla.edu \\ Jie Qing \\ Department of Mathematics \\ University of California \\ Santa Cruz, CA 95064 \\ qing@cats.ucsc.edu
}

These supporting institutions contribute to the cost of publication of this Journal, but they are not owners or publishers and have no responsibility for its contents or policies.

See inside back cover or msp.org/pjm for submission instructions.

The subscription price for 2013 is US \$400/year for the electronic version, and \$485/year for print and electronic.

Subscriptions, requests for back issues and changes of subscribers address should be sent to Pacific Journal of Mathematics, P.O. Box 4163, Berkeley, CA 94704-0163, U.S.A. The Pacific Journal of Mathematics is indexed by Mathematical Reviews, Zentralblatt MATH, PASCAL CNRS Index, Referativnyi Zhurnal, Current Mathematical Publications and Web of Knowledge (Science Citation Index).

The Pacific Journal of Mathematics (ISSN 0030-8730) at the University of California, c/o Department of Mathematics, 798 Evans Hall \#3840, Berkeley, CA 94720-3840, is published twelve times a year. Periodical rate postage paid at Berkeley, CA 94704, and additional mailing offices. POSTMASTER: send address changes to Pacific Journal of Mathematics, P.O. Box 4163, Berkeley, CA 94704-0163.

PJM peer review and production are managed by EditFLOW ${ }^{\circledR}$ from Mathematical Sciences Publishers.

\section{PUBLISHED BY}

mathematical sciences publishers

nonprofit scientific publishing

http://msp.org/

(C) 2013 Mathematical Sciences Publishers 


\section{PACIFIC JOURNAL OF MATHEMATICS}

Volume $266 \quad$ No. $2 \quad$ December 2013

Rate of attraction for a semilinear wave equation with variable coefficients and

critical nonlinearities

FÁGNER DiAs ARARUNA and FLANK DAVID MORAIS BEZERRA

The Brin-Thompson groups $s V$ are of type $\mathrm{F}_{\infty}$

Martin G. Fluch, Marco Marschler, Stefan Witzel and

MATTHEW C. B. ZAREMSKY

Ideal decompositions of a ternary ring of operators with predual

MASAYOSHI KANEDA

A study of real hypersurfaces with Ricci operators in 2-dimensional complex space 305 forms

Dong Ho Lim, WoOn Ha SoHn and Hyunjung Song

On commensurability of fibrations on a hyperbolic 3-manifold

HideTOSHI MASAI

Multiplicative Dirac structures

CRISTIÁN ORTIZ

On the finite generation of a family of Ext modules

TONY J. PuthenPuRAKAL

Index formulae for Stark units and their solutions

XAVIER-FranÇOIS ROBLOT

The short time asymptotics of Nash entropy

GUOYI XU

Several splitting criteria for vector bundles and reflexive sheaves

STEPHEN S.-T. YAU and FEI YE

The minimal volume orientable hyperbolic 3-manifold with 4 cusps

KEN'ICHI YOSHIDA

On the Witten rigidity theorem for string ${ }^{c}$ manifolds

JIANQING YU and BO LIU 